\title{
GROWTH OF SMALL GENERATING SETS IN $\mathrm{SL}_{n}(\mathbb{Z} / p \mathbb{Z})$
}

\author{
NICK GILL AND HARALD ANDRÉS HELFGOTT
}

\begin{abstract}
Let $G=\mathrm{SL}_{n}$ and fix $\delta$ a positive number. We prove that there are positive numbers $\epsilon$ and $C$ such that, for all fields $K=\mathbb{Z} / p \mathbb{Z}$ ( $p$ prime), and all sets $A \subset G(K)$ that generate $G(K)$, either $|A|<p^{n+1-\delta}, \delta>0$ or $|A \cdot A \cdot A| \geq C|A|^{1+\epsilon}$.
\end{abstract}

In $\left[\mathrm{He} 1\right.$ and $\mathrm{He} 2$, the second author proved that every set of generators of $\mathrm{SL}_{2}\left(\mathbb{F}_{p}\right)$ and $\mathrm{SL}_{3}\left(\mathbb{F}_{p}\right)$ grows rapidly. This has since found numerous applications $\left.([\mathrm{BG}],[\mathrm{BGS}], \mathrm{V}]\right)$ and generalisations to groups of the same type $\left(\mathrm{SL}_{2}, \mathrm{SL}_{3}\right)$ over other fields ([MCC], Di]). In the present paper, we prove that small subsets of $\mathrm{SL}_{n}\left(\mathbb{F}_{p}\right)$ grow.

Main Theorem. Let $G=\mathrm{SL}_{n}$ and fix $\delta$ a positive number. Then there are positive numbers $\epsilon$ and $C$ such that, for all fields $K=\mathbb{Z} / p \mathbb{Z}$ ( $p$ prime), and all sets $A \subset G(K)$ that generate $G(K)$, either $|A|<p^{n+1-\delta}, \delta>0$ or $|A \cdot A \cdot A| \geq C|A|^{1+\epsilon}$.

Here $|S|$ denotes the number of elements of a finite set $S$.

Our basic approach is to assume that $A$ is a set of generators for $G(K)$ that does not grow; i.e., for a fixed positive $\epsilon,|A \cdot A \cdot A| \ll_{n}|A|^{1+\epsilon}$. We will then show that if $A$ is "small" $\left(|A|<p^{n+1-\delta}\right)$ we have a contradiction with a result from arithmetic combinatorics (Prop. 5.2) related to the sum-product theorem and to incidence theorems.

\section{Notation AND BACKGROUnd}

We collect here the background ideas that will be needed in the sequel.

1.1. Arithmetic combinatorics. Our notation in this area is standard and, in particular, is identical to that of [He2]. In this section $G$ is an arbitrary group.

Given a positive integer $r$ and a subset $S$ of a group $G$, we define

$$
A_{r}=\left\{g_{1} \cdot g_{2} \cdots g_{r} \mid g_{i} \in A \cup A^{-1} \cup\{1\}\right\} .
$$

Given real numbers $a, b, x_{1}, \ldots, x_{n}$, we write

$$
a \ll_{x_{1}, \ldots, x_{n}} b
$$

to mean that the absolute value of $a$ is at most the real number $b$ multiplied by a constant $c$ depending only on $x_{1}, \ldots, x_{n}$. In this situation we also write $a=O_{x_{1}, \ldots, x_{n}}(b)$. When we omit $x_{1}, \ldots, x_{n}$, and write $a \ll b$, we mean that the constant $c$ is absolute, unless we explicitly state otherwise.

Occasionally we may write vectors in place of $x_{1}, \ldots, x_{n}$; for instance $a \ll \vec{v} b$ where $a$ and $b$ are real and $\vec{v}=\left(v_{1}, \ldots, v_{n}\right) \in \mathbb{R}^{n}$. In this case we mean that $a$ is at most the real number $b$ multiplied by a constant $c$ depending only on $v_{1}, \ldots, v_{n}$, and on $n$.

The next result will be useful in its own right; it also gives some more context to the statement of the Main Theorem. 
Lemma 1.1. [He2, Lem. 2.2] (Tripling Lemma). Let $k>2$ be an integer and let $c$ and $\epsilon$ be positive numbers. Then there exist positive numbers $c^{\prime}$ and $\epsilon^{\prime}$ such that, if $A$ is a finite subset of a group $G$ satisfying $\left|A_{k}\right| \geq c|A|^{1+\epsilon}$, then

$$
|A \cdot A \cdot A| \geq c^{\prime}|A|^{1+\epsilon^{\prime}}
$$

We include one final piece of notation: For an $n$-tuple $\left(x_{1}, \ldots, x_{n}\right)$, where $x_{1}, \ldots, x_{n}$ are elements of some set, we write $\left(x_{1}, \ldots, \widehat{x_{i}}, \ldots, x_{n}\right)$ to mean the $n-1$-tuple

$$
\left(x_{1}, \ldots, x_{i-1}, x_{i+1}, \ldots, x_{n}\right) \text {. }
$$

1.2. Degree and Escape. We will use the notion of escape from subvarieties in a very similar way to in [He2]. A full explanation of this idea is given in [He2]; we note here only some essential definitions and results.

For $V$ an affine algebraic variety, we define $\operatorname{dim} V$ to be the dimension of the irreducible subvariety of $V$ of largest dimension. As for the degree, it will be best to see it as a vector: we define the degree $\overrightarrow{\operatorname{deg}}(V)$ of an arbitrary variety $V$ to be

$$
\left(d_{0}, d_{1}, \ldots, d_{k}\right)
$$

where $k=\operatorname{dim}(V)$ and $d_{j}$ is the degree of the union of the irreducible components of $V$ of dimension $j$.

If a regular map $\phi: V \mapsto W$ between two varieties $V \subset \mathbb{A}^{m}, W \subset \mathbb{A}^{n}$ is defined by polynomials $\phi_{1}, \phi_{2}, \ldots, \phi_{n}$ on the variables $x_{1}, x_{2}, \ldots, x_{m}$, we define $\operatorname{deg}_{p o l}(\phi)$ to be $\max _{j} \operatorname{deg}\left(\phi_{j}\right)$. (If several representations of $\phi$ by polynomials $\phi_{1}, \phi_{2}, \ldots, \phi_{n}$ are possible, we choose - for the purposes of defining $\operatorname{deg}_{p o l}$ - the one that gives us the least value of $\operatorname{deg}_{p o l}$.)

Before proceeding we note an abuse of language: for a variety $V$ defined over a field $K$, and a subvariety $W$ defined over the algebraic completion $\bar{K}$ of $K$, we will write $W(K)$ for $W(\bar{K}) \cap V(K)$.

The following series of results is related to the idea of escape and non-singularity.

Proposition 1.2. [He2, Prop. 4.1] Let $G$ be a group. Consider a linear representation of $G$ on a vector space $\mathbb{A}^{n}(K)$ over a field $K$. Let $V$ be an affine subvariety of $\mathbb{A}^{n}$.

Let $A$ be a subset of $G$; let $\mathscr{O}$ be an $\langle A\rangle$-orbit in $\mathbb{A}^{n}(K)$ not contained in $V$. Then there are constants $\eta>0$ and $m$ depending only on $\overrightarrow{\operatorname{deg}}(V)$ such that, for every $x \in \mathscr{O}$, there are at least $\max (1, \eta|A|)$ elements $g \in A_{m}$ such that $g x \notin V$.

Lemma 1.3. [He2, Lem 4.3] Let $X \subseteq \mathbb{A}^{m_{1}}$ and $Y \subseteq \mathbb{A}^{m_{2}}$ be affine varieties defined over a field $K$. let $f: X \rightarrow Y$ be a regular map. Let $V$ be a subvariety of $X$ such that the derivative $\left.D f\right|_{x=x_{0}}$ of $f$ at $x=x_{0}$ is a nonsingular linear map for all $x_{0}$ on $X$ outside $V$.

Then, for any $S \subseteq X(\bar{K}) \backslash V(\bar{K})$, we have

$$
|f(S)| \gg \underset{\operatorname{deg}(X), \operatorname{deg}_{\mathrm{pol}}(f)}{ }|S| .
$$

Lemma 1.4. [He2, Lem.'s 4.4 and 4.6] Let $n$ be a positive integer, $\left(a_{1}, \ldots, a_{d}\right)$ and $\left(b_{1}, \ldots, b_{e}\right)$ be tuples of non-negative integers. There exist $c, d>0$ and a positive integer $k$ such that

- for all fields $K$ such that $|K|>c$;

- for all reductive algebraic groups $G \subset \mathrm{GL}_{n}$ defined over $K$ of degree $\left(a_{1}, \ldots, a_{e}\right)$; 
- for all subvarieties $V$ of $G$ of degree $\left(b_{1}, \ldots, b_{e}\right)$;

- for all $A \subset G(K)$ which generate $G(K)$;

we have

$$
\left|A_{k} \cap(G(K) \backslash V(K))\right| \geq d|A| .
$$

Corollary 1.5. [He2, Cor. 4.5] Let $G \subset \mathrm{GL}_{n}$ be an algebraic group of rank $r$ and $Y \subset \mathbb{A}^{m}$ an affine variety, both defined over a field $K$. Let $f: G \rightarrow Y$ be a regular map. Let $V$ be a proper subvariety of $G$. Assume that the derivative $\left.D f\right|_{x}$ of $f$ at $x$ is a nonsingular linear map for all $x$ on $G$ outside $V$.

Let $A \subset G(K)$ be a set of generators of $G(K)$. Then

$$
\left|f\left(A_{k} \cap(G(K) \backslash V(K))\right)\right| \gg \overrightarrow{\operatorname{deg}}(G), \overrightarrow{\operatorname{deg}}(V), \operatorname{deg}_{\mathrm{pol}}(f)|A|,
$$

where $k \ll \overrightarrow{\operatorname{deg}(V)} 1$.

Lemma 1.6. [He2, Lem. 4.7] Let $G \subseteq G L_{n}$ be an algebraic group defined over a field $K$. Let $X / K$ and $Y$ be a affine varieties such that $\operatorname{dim}(Y)=\operatorname{dim}(G)$. Let $f: X \times G \rightarrow Y$ be a regular map. Define

$$
f_{x}: G \rightarrow Y, g \mapsto f(x, g) .
$$

Then there is a subvariety $Z_{T \times G} \subset T \times G$ such that, for all $\left(x, g_{0}\right) \in(X \times G)(\bar{K})$, the derivative

$$
\left.\left(D f_{x}\right)\right|_{g=g_{0}}:\left.\left.(T G)\right|_{g=g_{0}} \rightarrow(T Y)\right|_{f\left(x, g_{0}\right)}
$$

is non-singular if and only if $\left(x, g_{0}\right)$ does not lie on $Z_{T \times G}$. Moreover,

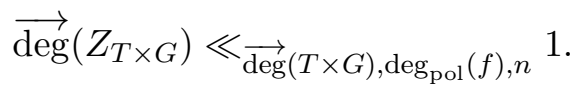

\section{KNOWN BOUNDS FOR SETS THAT DO NOT GROW.}

We set $G=S L_{n}, K=\mathbb{Z} / p \mathbb{Z}$; we use a number of results about generating sets in $G(K)$ that "do not grow" He2. We start with information about how such a set intersects with a torus of $G$.

Proposition 2.1. [He2, Cor.'s 5.4 and 5.10] Let $G=S L_{n}$.

(a) There exists $C>0$ and $k$ a positive integer such that, for all finite fields $K$ of characteristic $\neq 2$, for any $T$ a maximal torus of $G$ defined over $\bar{K}$, and for any $A \subset G(K)$ a set of generators of $G(K)$, we have $|A \cap T(K)| \leq C\left|A_{k}\right|^{\frac{1}{n+1}}$.

(b) There exist $d^{\prime}, \delta>0$ and $k^{\prime}$ a positive integer such that, for all finite fields $K$ of characteristic $\neq 2$, and for any $A \subset G(K)$ a set of generators of $G(K)$ satisfying $|A \cdot A \cdot A| \leq d|A|^{1+\epsilon}$ for some $d, \epsilon>0$, there is a maximal torus $T$ such that $\left|A_{k^{\prime}} \cap T(K)\right| \geq\left(d^{\prime} d^{\delta}\right)|A|^{\frac{1}{n+1}-\delta \epsilon}$.

A torus $T$ in $G$ that satisfies the last inequality will be called rich; that is to say, such a $T$ has a large intersection with the set $A_{k}$.

For a set $B \subset G(K)$ define $\operatorname{cl}(B)$ to be the set of conjugacy classes of $G(K)$ that intersect $B$; if $B$ contains only one element, $g$, then write $\operatorname{cl}(g)$ for $\operatorname{cl}(B)$. We also write $B^{\prime}$ for the set of semisimple elements in $B$; there are well-known bounds on $\left|\operatorname{cl}\left(A^{\prime}\right)\right|$ where $A$ is a generating set in $G(K)$ that does not grow. 
Proposition 2.2. [He2, Cor.'s 5.7 and 5.11] Let $G=S L_{n}$.

(a) There exists $C>0$ and a positive integer $k$ such that, for all finite fields $K$ of characteristic $\neq 2$, for any $T$ a maximal torus of $G$ defined over $\bar{K}$, and for any $A \subset G(K)$ a set of generators of $G(K)$, we have $\left|\operatorname{cl}\left(\left(A_{k}\right)^{\prime}\right)\right| \geq C|A|^{\frac{1}{n+1}}$.

(b) There exist $d^{\prime}, \delta>0$ and a positive integer $k^{\prime}$ such that, for all finite fields $K$ of characteristic $\neq 2$, and for any $A \subset G(K)$ a set of generators of $G(K)$ satisfying $|A \cdot A \cdot A| \leq d|A|^{1+\epsilon}$ for some $d, \epsilon>0$, we have $\left|\operatorname{cl}\left(A^{\prime}\right)\right| \leq\left(d^{\prime} d^{\delta}\right)\left|A_{k^{\prime}}\right|^{\frac{1}{n+1}+\delta \epsilon}$.

Note that the Tripling Lemma (Lem.1.1) implies that similar bounds apply to any set $A_{l}$ where $l \ll_{n} 1$; for instance, if $|A \cdot A \cdot A| \ll\left|A_{l}\right|^{1+\epsilon}$ and $l \ll_{n} 1$, then $\left|A_{l} \cdot A_{l} \cdot A_{l}\right| \ll\left|A_{l}\right|^{1+O_{l}(\epsilon)}$, and so

$$
\left|\operatorname{cl}\left(A_{l}^{\prime}\right)\right| \ll_{l, n}\left|A_{k l}\right|^{\frac{1}{n+1}+O_{l, n}(\epsilon)} \ll_{l, n}|A|^{\frac{1}{n+1}+O_{l, n}(\epsilon)} .
$$

We need to be sure that the intersection of $A_{k_{\dagger}}$ with a rich torus $T$ does not lie inside a subtorus (of a certain kind).

Proposition 2.3. [He2, Cor. 5.14] Let $G=S L_{n}$ and let $\left(a_{1}, \ldots, a_{r}\right)$ be a tuple of nonnegative integers. Then there exists $C>0$ and a positive integer $k$ such that for a finite field $K$ with characteristic $>n$; for $T$ a maximal torus of $G$ defined over $\bar{K}$; for $\alpha: T \rightarrow \mathbb{A}^{1}$ a character of $T$ with kernel $T^{\prime}$ satisfying $\operatorname{deg}\left(T^{\prime}\right)=\left(a_{1}, \ldots, a_{r}\right)$; and for $A \subset G(K)$ a set of generators of $G(K)$, we have

$$
\left|A \cap T^{\prime}(K)\right| \leq C\left|A_{k}\right|^{\frac{1}{n+2}} .
$$

The significance of this special type of subtorus is explained by the next result.

Lemma 2.4. [He2, Lem.'s 4.2 and 4.14] Let $K$ be a field. Let $T / \bar{K} \subset\left(\mathrm{GL}_{1}\right)^{n}$ be a torus. Let $H$ be a subgroup of $T(\bar{K})$ that is contained in an algebraic subvariety $V$ of $T$ of positive codimension.

Then $H$ is contained in the kernel of a non-trivial character $\alpha: T \rightarrow G L_{1}$ whose exponents are bounded in terms of $n$ and $\overrightarrow{d e g}(V)$ alone.

2.1. Further results on conjugacy. We need several more ideas concerning conjugacy classes in $G$. An element $g$ of a linear algebraic group $G$ is said to be regular if $\operatorname{dim}\left(C_{G}(g)\right)$ is equal to the rank of $G$. When $G$ is connected, and $g$ is semisimple this is equivalent to requiring that $C_{G}(g)$ is a torus. When $G=S L_{n}$, an element is regular semisimple if and only if its eigenvalues are distinct.

Lemma 2.5. [He2, Lem. 5.9] Let $G=S L_{n}$ and $K$ be a field. Then there is a subvariety $W / K$ of $G$ of positive codimension and degree $\overrightarrow{\operatorname{deg}}(W)=\left(a_{1}, \ldots, a_{d}\right)$ such that $a_{1}, \ldots a_{d}, d<n^{2 n}$, and every element $g \in G(K)$ not on $W$ is regular semisimple.

Of course, since $W$ is a subvariety of $S L_{n}$ of positive codimension, we may apply the results of $₫ 1.2$, and escape from it. Escaping from a subvariety $W$ of a torus is only slightly harder, provided that the torus is rich.

Lemma 2.6. Let $G=S L_{n}$ and let $\left(w_{1}, \ldots, w_{d}\right)$ be a tuple of non-negative integers. There exists a positive integer $l$ and $\epsilon_{0}, c>0$ such that

(a) for all finite fields $K$ of characteristic $>n$; 
(b) for any $A$ a set of generators for $G$ satisfying $|A \cdot A \cdot A| \leq d|A|^{1+\epsilon}$ for some $d, \epsilon>0$ with $\epsilon<\epsilon_{0}$;

(c) for any corresponding rich torus $T\left(\right.$ so $\left.\left|A_{k} \cap T(K)\right| \geq\left(d^{\prime} d^{\delta}\right)|A|^{\frac{1}{n+1}-\delta \epsilon}\right)$;

(d) for any $W$ a proper subvariety of $T$ of degree $\left(w_{1}, \ldots, w_{d}\right)$;

we have

$$
\left|A_{l} \cap(T(K) \backslash W(K))\right| \geq c|A|^{\frac{1}{n+1}-\delta \epsilon} .
$$

Note that the value of $\delta$ given in the conclusion to Lem. 2.6 is fixed by Prop. 2.1 (as per the inequality written above at (ㄷ) ).

Proof. Suppose first that $\left\langle A_{l} \cap T(K)\right\rangle$ is not contained in $W(K)$. Then apply Prop. 1.2 with $A_{l} \cap T(K)$ instead of $A$, and $W$ instead of $V$, and the result follows.

Now suppose that $\left\langle A_{l} \cap T(K)\right\rangle \subseteq W(K)$. Then, by Lem. 2.4. $\left\langle A_{l} \cap T(K)\right\rangle$ is contained in the kernel of a non-trivial character $\alpha: T \rightarrow \mathbb{A}^{1}$ whose exponents are bounded in terms of $n$ and $\overrightarrow{\operatorname{deg}}(W)$ alone. Hence, by Prop. 2.3 with $A_{l}$ instead of $A$, and $l^{\prime}$ instead of $k$,

$$
\left|A_{l} \cap T(K)\right| \ll_{n, \overrightarrow{\operatorname{deg}}(W)}\left|A_{l l^{\prime}}\right|^{\frac{1}{n+2}} \ll|A|^{\frac{1}{n+2}+O_{n}(\epsilon)} .
$$

This contradicts the assumption that $T$ is a rich torus.

In what follows, we fix $\epsilon>0$, and fix $k_{\dagger}=\max \left\{k, k^{\prime}, l\right\}$ where $k$ and $k^{\prime}$ are as given in Prop. 2.2, and $l$ is as given in Lemma 2.6, for the latter result we take $W$ to be the variety defined in Lemma 2.5. Thus, for $A \subset G(K)$ a generating set that doesn't grow,

and for $T$ a rich torus, we have tight bounds on the intersection of $A_{k_{\dagger}}$ with the set of regular elements in $T(K)$, as well as on the number of regular conjugacy classes in $A_{k_{\dagger}}$.

Our analysis of conjugacy classes in $G$ will require one more definition: Given a matrix $g$ in $\mathrm{SL}_{n}(K)$, we define $\kappa(g) \in \mathbb{A}^{n-1}(K)$ to be the tuple

$$
\left(a_{n-1}, a_{n-2}, \ldots, a_{1}\right)
$$

of coefficients of

$$
\lambda^{n}+a_{n-1} \lambda^{n-1}+a_{n-2} \lambda^{n-2}+\ldots+a_{1} \lambda+(-1)^{n}=\operatorname{det}(\lambda I-g) \in K[\lambda]
$$

(the characteristic polynomial of $g$ ).

As is well-known, $\kappa(g)=\kappa\left(h g h^{-1}\right)$ for any $h$, i.e., $\kappa(g)$ is invariant under conjugation. If $g$ is a regular semisimple element of $\mathrm{SL}_{n}$ then $\kappa(g)$ actually determines the conjugacy class $\operatorname{cl}(g)$ of $g$.

\section{A NEW BOUnd ON SETS THAT DO NOT GROW}

Just as in Helfgott's work on $S L_{3}$, the trace is going to be important for us, although we will use it in a very different way.

3.1. Independence of traces. In this section we show that a certain set of linear forms is linearly dependent, and that any subset is linearly independent.

Let $m \in K\left[t_{1}, \ldots, t_{n}\right]$ be a monomial; we call $m$ square-free if $t_{j}^{2}$ does not divide $m$ for any $j=1, \ldots, n$. 
Lemma 3.1. 1 Let $s_{j}, j=1, \ldots, n$, be distinct elements of $K^{*}$. Then, for any $i, 0 \leq i \leq n$,

$$
A_{i}=\left(\begin{array}{ccccccc}
1 & s_{1} & \cdots & s_{1}^{i-1} & s_{1}^{i+1} & \cdots & s_{1}^{n} \\
1 & s_{2} & \cdots & s_{2}^{i-1} & s_{2}^{i+1} & \cdots & s_{2}^{n} \\
\vdots & \vdots & & \vdots & \vdots & & \vdots \\
1 & s_{n} & \cdots & s_{n}^{i-1} & s_{n}^{i+1} & \cdots & s_{n}^{n}
\end{array}\right)
$$

has determinant equal to

$$
\left(\prod_{1 \leq j<k \leq n}\left(s_{k}-s_{j}\right)\right) \times S_{n-i}
$$

where $S_{n-i}$ is the sum of all square-free monomials of degree $n-i$ in the variables $s_{1}, \ldots, s_{n}$. Proof. We prove the result by calculating a Vandermonde determinant in $\left\{s_{1}, \ldots, s_{n}, t\right\}$ for some variable $t$. We define

$$
f=\operatorname{det}\left(\begin{array}{cccc}
1 & s_{1} & \cdots & s_{1}^{n} \\
1 & s_{2} & \cdots & s_{2}^{n} \\
\vdots & \vdots & & \vdots \\
1 & s_{n} & \cdots & s_{n}^{n} \\
1 & t & \cdots & t^{n}
\end{array}\right)
$$

and consider $f$ as a polynomial of degree $n$ in $t$. Observe that $\operatorname{det} A_{i}=(-1)^{n+i} c_{i}$ where $c_{i}$ is the coefficient of $t^{i}$ in $f$.

The classical formula for a Vandermonde determinant implies that

$$
f=\prod_{1 \leq j<k \leq n}\left(s_{k}-s_{j}\right) \prod_{1 \leq k \leq n}\left(t-s_{k}\right) .
$$

Then $f=\left(\prod_{1 \leq j<k \leq n}\left(s_{k}-s_{j}\right)\right) g$ where $g=\prod_{1 \leq k \leq n}\left(t-s_{k}\right)$ is a polynomial of degree $n$ in $t$. Write $d_{i}$ for the coefficient of $t^{i}$ in $g$; to prove the lemma it is sufficient to observe that $d_{i}=(-1)^{n-i} S_{n-i}$.

Lemma 3.2. 2 Suppose that $t$ is a regular element of $T(K)$. For $i=0, \ldots, n$, define linear forms,

$$
l_{i}: G(K) \rightarrow K: g \mapsto \operatorname{tr}\left(t^{i} g\right) .
$$

These forms are linearly dependent. Furthermore, there exists a subvariety $W$ in $T$ of positive codimension and degree $\ll_{n} 1$, such that if $t \in T(K) \backslash W(K)$, then any subset of $n$ of these linear forms is linearly independent.

Proof. Write $T(K)$ as the group of $n \times n$ diagonal matrices over $K$ of determinant 1 . The linear forms $l_{i}$ are forms on $n$ variables, $g_{11}, \ldots, g_{n n}$. Since there are $n+1$ of these, they must be linearly dependent.

\footnotetext{
${ }^{1}$ We thank an anonymous referee for significantly simplifying the proof of this result.

${ }^{2} \mathrm{An}$ anonymous referee pointed out that this result follows directly from the Cayley-Hamilton identity; c.f. the proof of Lemma 5.1 .
} 
Now consider a set, $L$, of $n$ of these linear forms. Since $t$ is regular, all eigenvalues are distinct. Thus $L$ will be linearly independent provided a matrix of the form given in Lemma 3.1 is non-zero.

For $i, j=1, \ldots, n$ with $i<j$ define $V_{i, j}$ to be the variety defined by $g_{i i}-g_{j j}=0$, and let $V_{-1}=\bigcup_{1 \leq i<j \leq n} V_{i, j}$. Thus $V(K)$ contains all elements of $T(K)$ that are not regular.

Next define $W_{i}$ to be the variety defined by the equation $S_{i}=0$, where $S_{i}$ is as in Lemma 3.1] let $W_{-1}=\cup_{i=0}^{n} W_{i}$ and let $W=V_{-1} \cup W_{-1}$.

Clearly $V_{i, j}, i, j=1, \ldots, n$ with $i<j$, and $W_{i}, i=0, \ldots, n$ are subvarieties of $T$ of positive codimension. Then Lemma 3.1 yields the result.

3.2. A new bound. Now we work towards proving a new bound on generating sets that "do not grow." We begin with some notation. Fix $t$ an element of a torus $T(K)$ in $G(K)$, $i$ some integer satisfying $0 \leq i \leq n$. For $g$ an element of $G(K)$, define two $n$-tuples as follows:

$$
\begin{aligned}
\operatorname{Tr}_{\widehat{i}, t}(g) & =\left(\operatorname{tr}(g), \operatorname{tr}(t g), \ldots, \widehat{\operatorname{tr}\left(t^{i} g\right)}, \ldots, \operatorname{tr}\left(t^{n} g\right)\right) ; \\
\mathrm{Cl}_{\hat{i}, t}(g) & =\left(\operatorname{cl}(g), \operatorname{cl}(t g), \ldots, \widehat{\operatorname{cl}\left(t^{i} g\right)}, \ldots, \operatorname{cl}\left(t^{n} g\right)\right)
\end{aligned}
$$

For a set $A \subset G(K)$, define

$$
\begin{aligned}
& \operatorname{Tr}_{\widehat{i}, t}(A)=\left\{\operatorname{Tr}_{\widehat{i}, t}(g) \mid g \in A\right\} \\
& \mathrm{Cl}_{\hat{i}, t}(A)=\left\{\mathrm{Cl}_{\widehat{i}, t}(g) \mid g \in A\right\} \\
& \mathrm{Cl}_{\hat{i}, t}^{\prime}(A)=\left\{\mathrm{Cl}_{\widehat{i}, t}(g) \mid g \in A, \text { and } g, t g, \ldots, t^{n} g \text { are semisimple }\right\} .
\end{aligned}
$$

The inequality that we will prove relates to $\mathrm{Cl}_{\hat{i}, t}(A)$. In fact $\mathrm{Cl}_{\hat{i}, t}(A)$ corresponds (generically) to $\mathrm{cl}_{T(K)}(A)$, the set of $T(K)$-conjugacy classes of $G(K)$ that intersect $A$. We will not need this correspondence in what follows.

Lemma 3.3. Let $G=S L_{n}$, and let $K$ be a field. For $h_{0}, t_{0}, \ldots, t_{n} \in G(\bar{K})$, define

$$
f_{h_{0}, t_{0}, \ldots, t_{n}}: G \rightarrow \mathbb{A}^{n^{2}-1}: g \mapsto\left(\kappa\left(h_{0} g\right), \kappa\left(t_{0} g\right), \ldots, \kappa\left(t_{n} g\right)\right) .
$$

Define

$$
f_{\widehat{i}, h_{0}, t_{0}, \ldots, t_{n}}: G \rightarrow \mathbb{A}^{n^{2}-n}: g \mapsto\left(\kappa\left(h_{0} g\right), \kappa\left(t_{0} g\right), \ldots, \widehat{\kappa\left(t_{i} g\right)}, \ldots \kappa\left(t_{n} g\right)\right)
$$

Let $T / \bar{K}$ be a maximal torus of $G$. Then there exists $h_{0} \in G(\bar{K}), t \in T(\bar{K})$, and $g_{0} \in$ $G(\bar{K})$, such that the derivative of $f_{\widehat{i}, h_{0}, t, t^{2} \ldots, t^{n}}$ at $g=g_{0}$ is a nonsingular linear map.

This result should be compared with [He2, Lemma 5.5]; the method of proof is very similar. 
Proof. We may write the elements of $G(\bar{K})$ so that the elements of $T(\bar{K}) \subset G(\bar{K})$ become diagonal matrices. Let

$$
g_{0}=\left(\begin{array}{cccccc}
0 & 1 & 0 & \cdots & 0 & 0 \\
0 & 0 & 1 & \cdots & 0 & 0 \\
\vdots & \vdots & \vdots & \vdots & \vdots & \vdots \\
0 & 0 & 0 & \cdots & 1 & 0 \\
0 & 0 & 0 & \cdots & 0 & 1 \\
(-1)^{n-1} & 0 & 0 & \cdots & 0 & 0
\end{array}\right)
$$

Let $\vec{r}=\left(r_{1}, r_{2}, \ldots, r_{n}\right)$ be a vector in $\bar{K}^{n}$ with $r_{1} \cdot r_{2} \cdots r_{n}=1$. Define

$$
h_{1}=\left(\begin{array}{cccc}
r_{1} & 0 & \ldots & 0 \\
0 & r_{2} & \ldots & 0 \\
\vdots & \vdots & \vdots & \vdots \\
0 & 0 & \ldots & r_{n}
\end{array}\right)
$$

Let us look, then, at the derivative at $g=I$ of $g \mapsto \kappa\left(h_{1}^{j} g_{0} g\right)$ for $0 \leq j \leq n$. The derivative at $g=I$ of the map taking $g$ to the coefficient of $\lambda^{n-1}$ in $\operatorname{det}\left(\lambda I-h_{1}^{j} g_{0} g\right)$ (i.e., to $(-1)$ times the trace of $\left.h_{1}^{j} g_{0} g\right)$ is equal to the map taking each matrix $\gamma$ in the tangent space $\mathfrak{g}$ to $G$ at the origin to

$$
(-1) \cdot\left(r_{1}^{j} \gamma_{2,1}+r_{2}^{j} \gamma_{3,2}+\ldots+r_{k}^{j} \gamma_{k+1, k}+\ldots+(-1)^{n-1} r_{n}^{j} \gamma_{1, n}\right),
$$

where we write $\gamma_{k, l}$ for the entries of the matrix $\gamma$.

The derivative at $g=I$ of the map taking $g$ to the coefficient of $\lambda^{n-2}$ in $\operatorname{det}\left(\lambda I-h_{1}^{j} g_{0} g\right)$ is the map taking each $\gamma$ in $\mathfrak{g}$ to

$$
\begin{aligned}
(-1) \cdot\left(r_{1}^{j} r_{2}^{j} \gamma_{3,1}\right. & +r_{2}^{j} r_{3}^{j} \gamma_{4,2}+\ldots+r_{k}^{j} r_{k+1}^{k} \gamma_{k+2, k}+\ldots+r_{n-3}^{j} r_{n-2}^{j} \gamma_{n-1, n-3} \\
& \left.+r_{n-2}^{j} r_{n-1}^{j} \gamma_{n, n-2}+r_{n-1}^{j} \cdot(-1)^{n-1} r_{n}^{j} \gamma_{1, n-1}+(-1)^{n-1} r_{n}^{j} r_{1}^{j} \gamma_{2, n}\right) .
\end{aligned}
$$

In general, for $1 \leq l \leq n-1$, the derivative at $g=I$ of the map taking $g$ to the coefficient of $\lambda^{n-l}$ in $\operatorname{det}\left(\lambda I-h_{1}^{j} g_{0} g\right)$ is the map taking $\gamma$ to

$$
(-1) \sum_{k=1}^{n-k}\left(r_{k}^{j} \cdot r_{\underline{k+1}}^{j} \cdots r_{\underline{k+l-1}}^{j}\right) \cdot \gamma_{\underline{k+l, k}}+(-1)^{n} \sum_{k=n-l+1}^{n}\left(r_{k}^{j} \cdot r_{\underline{k+1}}^{j} \cdots r_{\underline{k+l-1}}^{j}\right) \cdot \gamma_{\underline{k+l, k}}
$$

where by $\underline{a}$ we mean the only element of $\{1,2, \ldots, n\}$ congruent to $a$ modulo $n$.

We see that the entries of $\gamma$ present in (3.2) are disjoint for distinct $1 \leq l \leq n-1$ (and disjoint from $\left\{\gamma_{1,1}, \gamma_{2,2}, \ldots, \gamma_{n, n}\right\}$, which would appear for $l=0$ ). Now, for $l$ fixed, (3.2) gives us a linear form on $n$ variables $\gamma_{k+l, k}$ for each $\leq j \leq n$. Let us check that, for every $1 \leq l \leq n-1$, any $n$ of these linear forms are linearly independent, provided that $\vec{r}$ was chosen correctly.

This is the same as checking that the $n-1$ determinants

$$
\left|\left(r_{k}^{j} \cdot \underline{r_{k+1}^{j}} \cdots r_{\underline{k+l-1}}^{j}\right)\right|_{1 \leq k \leq n, 0 \leq j \leq n, j \neq i}
$$

for $1 \leq l \leq n-1$ are non-zero for some choice of $r_{1}, r_{2}, \ldots, r_{n}$ with $r_{1} \cdot r_{2} \cdots r_{n}=1$. (What we really want to check is that the determinant (3.3) is non-zero after all signs in some 
columns are flipped; since those flips do not affect the absolute value of the determinant, it is just as good to check that the determinant (3.3) itself is non-zero.)

These are generalised Vandermonde determinants of the type studied in Lemma 3.1. that result implies that they have absolute value equal to

$$
\prod_{k_{1}<k_{2}}\left(r_{k_{2}} \cdot \underline{r_{k_{2}+1}} \cdots \underline{r_{k_{2}+l-1}}-r_{k_{1}} \cdot r_{\underline{k_{1}+1}} \cdots r_{\underline{k_{1}+l-1}}\right) \times S_{n-i}
$$

where $S_{n-i}$ is the sum of all square-free monomials $m$ of degree $n-i$ in the variables $r_{k} \cdot r_{k+1} \cdots r_{k+l-1}$ for $k=1, \ldots, n$.

For any given $k_{1}, k_{2} \in\{1, \ldots, n\}$ with $k_{1} \neq k_{2}$, and $l \in\{1, \ldots, n-1\}$, there exist $r_{1}, r_{2}, \ldots, r_{n} \in \bar{K}$ with $r_{1} r_{2} \cdots r_{n}=1$ such that $r_{k_{1}} \cdot r_{k_{1}+1} \cdots r_{k_{1}+l-1} \neq r_{k_{2}} \cdot r_{k_{2}+1} \cdots r_{k_{2}+l-1}$.

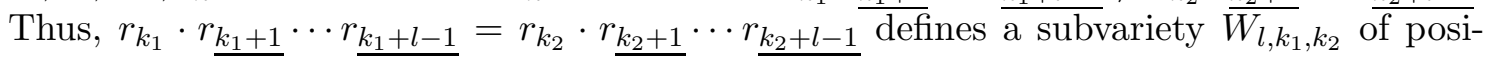
tive codimension in the (irreducible) variety $\overline{V \subset} \mathbb{A}^{n}$ of all tuples $\left(r_{1}, r_{2}, \cdots, r_{n}\right)$ with $r_{1} r_{2} \cdots r_{n}=1$.

In addition, for $i, j=1, \ldots, n$ with $i<j$, define $V_{i, j}$, the subvariety of $V$ given by the equation $r_{i}=r_{i}$; clearly the set $V \backslash V_{i, j}(\bar{K})$ is non-empty, and so these varieties are also of positive codimension in $V$. Then

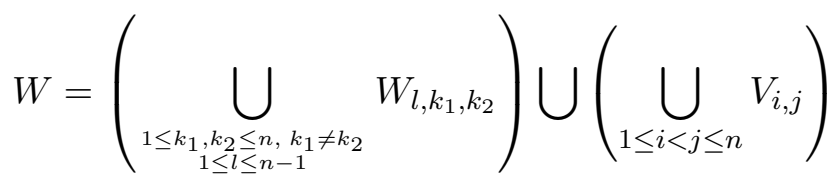

is a finite union of subvarieties of $V$ of positive codimension. Take $\vec{r}$ to be any point of $V(\bar{K})$ outside $W(\bar{K})$. It remains to choose $h_{0}$ so that the derivative of

$$
g \mapsto \kappa\left(h_{0} g\right)
$$

at $g=I$ is a linear map of full rank on the diagonal entries $\gamma_{1,1}, \gamma_{2,2} \ldots, \gamma_{n-1, n-1}$ of $\mathfrak{g}$. Let

$$
h_{0}=\left(\begin{array}{cccc}
t_{1} & 0 & \ldots & 0 \\
0 & t_{2} & \ldots & 0 \\
\vdots & \vdots & \vdots & \vdots \\
0 & 0 & \ldots & t_{n}
\end{array}\right),
$$

where $t_{1}, t_{2}, \ldots, t_{n} \in \bar{K}$ fulfil $t_{1} t_{2} \cdots t_{n}=1$. Then the derivative at $g=I$ of the map taking $g$ to the coefficient of $\lambda^{n-1}$ in $\operatorname{det}\left(\lambda I-h_{0} g\right.$ ) (i.e., to $(-1)$ times the trace of $h_{0} g$ ) equals the map taking $\gamma$ to

$$
(-1) \cdot\left(t_{1} \gamma_{1,1}+t_{2} \gamma_{2,2}+\cdots+t_{n} \gamma_{n, n}\right) .
$$

In general, the derivative of the map taking $g$ to the coefficient of $\lambda^{n-k}(1 \leq k \leq n-1)$ in $\operatorname{det}\left(\lambda I-h_{0} g\right)$ equals the map taking $\gamma$ to

$$
(-1)^{k} \cdot\left(c_{k, 1} \gamma_{1,1}+c_{k, 2} \gamma_{2,2}+\cdots+c_{k, n} \gamma_{n, n}\right)
$$

where $c_{k, i}$ is the sum of all monomials $t_{j_{1}} t_{j_{2}} \ldots t_{j_{k}}, 1 \leq j_{1}<j_{2}<\cdots<j_{k} \leq n$, such that one of the indices $j_{l}$ equals $i$. (For example, $c_{2,1}=t_{1} \cdot\left(t_{2}+t_{3}+\cdots+t_{n}\right)$.) Thus, our task is to find for which $t_{1}, t_{2}, \ldots, t_{n}$ the determinant

$$
\left|c_{i, j}-c_{i, n}\right|_{1 \leq i, j \leq n-1}
$$


is non-zero. Clearly, this will happen precisely when

$$
\left|c_{i-1, j}\right|_{1 \leq i, j \leq n} \neq 0,
$$

where we adopt the (sensible) convention that $c_{0, j}=1$ for all $j$.

A brief computation gives us that

$$
\left|c_{i-1, j}\right|_{1 \leq i, j \leq n}=(-1)^{\lfloor n / 2\rfloor} \cdot\left|t_{j}^{i-1}\right|_{1 \leq i, j \leq n} .
$$

This is a Vandermonde determinant; it equals $\prod_{j_{1}<j_{2}}\left(t_{j_{2}}-t_{j_{1}}\right)$. The equation $t_{j_{2}}=t_{j_{1}}$ defines a subvariety of positive codimension in the variety $V \subset \mathbb{A}^{n}$ of all $t_{1}, t_{2}, \ldots, t_{n}$ with $t_{1} t_{2} \cdots t_{n}=1$. Thus, we may choose $t_{1}, t_{2}, \ldots, t_{n}$ such that $t_{1} t_{2} \cdots t_{n}=1$ and $\prod_{j_{1}<j_{2}}\left(t_{j_{2}}-t_{j_{1}}\right) \neq 0$.

We are now able to prove the proposition that we have been aiming for (c.f. [He2, Prop. 5.15]).

Proposition 3.4. Let $G=\mathrm{SL}_{n}$. There exists a positive integer $k$ and $c, e>0$ such that for any $\left(a_{1}, \ldots, a_{d}\right)$, a tuple of non-negative integers, there exists a positive integer $k^{\prime}$ and $c^{\prime}>0$ such that

- for any $K$ a finite field such that $|K|<e$;

- for any $W / \bar{K}$, a proper subvariety of $G$, such that $\overrightarrow{d e g}(W)=\left(a_{1}, \ldots, a_{d}\right)$;

- for any $E \subset T(K)$ where $T / \bar{K}$ is a maximal torus of $G$.

- for any $A \subset G(K)$, a set of generators of $G(K)$; ;

one of the following holds

(a) there exists $h_{0} \in A_{k}$, and a subset $E^{\prime} \subset E_{k}$ with $\left|E^{\prime}\right| \geq c|E|$ such that, for each $t \in E^{\prime}$, there are $\geq c^{\prime}|A|$ distinct tuples

$$
\left(\kappa\left(h_{0} g\right), \kappa(g), \kappa(t g), \kappa\left(t^{2} g\right), \ldots, \widehat{\kappa\left(t^{i} g\right)}, \ldots, \kappa\left(t^{n} g\right)\right) \in \mathbb{A}^{n^{2}-1}(K)
$$

with $g \in A_{k^{\prime}}$ satisfying $h_{0} g, g, t g, t^{2} g, \ldots, t^{n} g \notin W(K)$, or

(b) $E$ is contained in the kernel of a non-trivial character $\alpha: T \rightarrow G L_{1}$ whose exponents are bounded in terms of $n$ alone.

Proof. Let $X=G \times T$ and $Y=\left(\mathbb{A}^{n-1}\right)^{n+1}=\mathbb{A}^{n^{2}-1}$. Let $f: X \times G \rightarrow Y$ be given by

$$
f\left(\left(h_{0}, t\right), g\right)=\left(\kappa(h g), \kappa(g), \kappa(t g), \kappa\left(t^{2} g\right), \ldots, \widehat{\kappa\left(t^{i} g\right)}, \ldots, \kappa\left(t^{n} g\right)\right) .
$$

Let $Z_{X \times G}$ be as in Lemma 1.6, then $\overrightarrow{\operatorname{deg}}\left(Z_{X \times G}\right) \ll_{n} 1$. Thanks to Lem. 3.3, we know $Z_{X \times G}$ is a proper subvariety of $X \times G$.

Let $Z_{G \times T \times G}$ be $Z_{X \times G}$ under the identification $G \times T \times G=X \times G$; write the elements of $Z_{G \times T \times G}$ in the form $\left(h_{0}, t, g\right)$. By the argument in [He2, $\left.\S 2.5 .3\right]$, there is a proper subvariety $Z_{G} \subset G$ (with $\overrightarrow{\operatorname{deg}}\left(Z_{G}\right) \ll \overrightarrow{\operatorname{deg}}\left(Z_{G \times T \times G}\right)$, and so $\overrightarrow{\operatorname{deg}}\left(Z_{G}\right) \ll_{n} 1$ ) such that, for all $h_{0} \in G(\bar{K})$ not on $Z_{G}$, the fibre $\left(Z_{G \times T \times G}\right)_{h=h_{0}}$ is a proper subvariety of $T \times G$.

By escape (Lem. 1.4, it is here that that $|K| \gg_{n} 1$ is used), there is an $h_{0} \in A_{k}, k \ll_{n} 1$, such that $h_{0}$ lies outside $Z_{G}$; thus, by the definition of $Z_{G}$, the fibre $V_{T \times G}:=\left(Z_{G \times T \times G}\right)_{h=h_{0}}$ is a proper subvariety of $T \times G$. Again by [He2, $\S 2.5 .3]$, there is a proper subvariety $V_{T}$ with $\overrightarrow{\operatorname{deg}}\left(V_{T}\right) \ll \overrightarrow{\operatorname{deg}}\left(V_{T \times G}\right) 1$ (and so $\overrightarrow{\operatorname{deg}}\left(V_{T}\right) \ll_{n} 1$ ) such that, for all $t_{0} \in T(\bar{K})$ not on $V_{T}$, the fibre $\left(V_{T \times G}\right)_{t=t_{0}}$ is a proper subvariety of $G$. 
Suppose first that $\langle E\rangle \not \subset V_{T}(K)$. We may then use escape from subvarieties (Prop. 1.2 with $A=E, V=V_{T}(K)$ and $\left.G=\mathscr{O}=\langle E\rangle\right)$ to obtain a subset $E^{\prime} \subset E_{k}\left(k \ll_{n} 1\right)$ with $\left|E^{\prime}\right| \gg_{n}|E|$ and $E^{\prime} \subset T(K) \backslash V_{T}(K)$. Now consider any $t_{0} \in E^{\prime}$. The fibre $\left(V_{T \times G}\right)_{t=t_{0}}$ is a proper subvariety of $G$, and, since $W$ is a proper subvariety of $G$, we conclude that

$$
V^{\prime}=\left(V_{T \times G}\right)_{t=t_{0}} \cup h_{0}^{-1} W \cup W \cup t_{0}^{-1} W \cup \ldots \cup t_{0}^{-n} W .
$$

is a proper subvariety of $G$ as well (with $\overrightarrow{\operatorname{deg}}\left(V^{\prime}\right) \ll_{n, \overrightarrow{\operatorname{deg}}(W)} 1$ ). We now recall the definition of $Z_{X \times G}$ (a variety outside which the map $f$ is non-singular) and use the result on non-singularity (Cor. 1.5 applied to the function $f_{h_{0}, t_{0}}: G \rightarrow Y$ given by $\left.f_{h_{0}, t_{0}}(g)=f\left(\left(h_{0}, t_{0}\right), g\right)\right)$ to obtain that

$$
\left|f_{h_{0}, t_{0}}\left(A_{k^{\prime}} \cap\left(G(K) \backslash V^{\prime}(K)\right)\right)\right| \gg_{n, \overrightarrow{\operatorname{deg}}(W)}|A|
$$

with $k^{\prime} \ll_{n, \overrightarrow{\operatorname{deg}}(W)} 1$. This gives us conclusion (国).

Suppose now that $\langle E\rangle \subset V_{T}(K)$. By Lemma 2.4, we obtain that $E$ is contained in the kernel of a non-trivial character $\alpha: T \rightarrow \mathbb{A}^{1}$ whose exponents are $\ll_{n} 1$.

Given our generating set $A$ satisfying $|A \cdot A \cdot A| \ll_{n}|A|^{1+\epsilon}$, recall that Prop. 2.1 implies that there exists a torus $T$ that is rich. In other words

$$
\left|A_{k} \cap T(K)\right| \gg|A|^{\frac{1}{n+1}}-O_{n}(\epsilon)
$$

for some $k \ll_{n} 1$. In fact, by Lem. 2.6 we know that when $T$ is rich, the set of regular elements in $A_{k_{\dagger}} \cap T(K)$ has size $\gg|A|^{\frac{1}{n+1}}-O_{n}(\epsilon)$, where $k_{\dagger} \ll_{n} 1$. These facts allow us to prove the following corollary.

Corollary 3.5. Let $\epsilon>0$ and suppose that $\epsilon$ is smaller than some constant $\epsilon_{0}$ depending only on $n$. Let $A$ be a generating set such that $|A \cdot A \cdot A| \ll_{n}|A|^{1+\epsilon}$, and let $T$ be a rich maximal torus. Then there are integers $k, l \ll_{n} k_{\dagger}$ and a set $E^{\prime}$ in $A_{k} \cap T(K)$ such that $\left|E^{\prime}\right| \gg|A|^{\frac{1}{n+1}+O_{n}(\epsilon)}$ and

$$
|A|^{\frac{n}{n+1}-O_{n}(\epsilon)} \ll_{n}\left|\mathrm{Cl}_{i, t}^{\prime}\left(A_{l}\right)\right|,
$$

for all $t \in E^{\prime}$. Moreover there are $\gg|A|$ elements of $A_{l}$ such that $g, t g, \ldots, t^{n} g$ are semisimple.

Proof. Let $W$ be the variety of elements in $G$ that are not semisimple, and note that, by Lem. 2.5, $\overrightarrow{\operatorname{deg}}(W) \ll_{n} 1$. Set $E=A_{k_{\dagger \dagger}} \cap T(K)$ where $T$ is a rich torus and $k_{\dagger \dagger}=\max \left\{k_{\dagger}, k\right\}$ for $k$ the constant given in Prop. 3.4, thus Prop. 2.1 implies that $|E| \gg_{n}|A|^{\frac{1}{n+1}-O_{n}(\epsilon)}$. Now apply Prop. 3.4 to $A$. Since we are assuming that $|A \cdot A \cdot A| \ll_{n}|A|^{1+\epsilon}$, we may assume that $K$ is larger than some constant depending only on $n$ (otherwise our assumption is trivially violated). Thus we lie in situation (a) or (b) of Prop. 3.4.

Suppose first that we are in situation (b). Then Lem. 2.4 and Prop. 2.3 imply that $|E| \ll_{n}\left|A_{l}\right|^{\frac{1}{n+2}}$ where $l \ll_{n} 1$. For $\epsilon$ small enough with respect to $n$ this gives a contradiction.

Thus we are in situation (a) and observe first that

$$
\left|E^{\prime}\right| \gg_{n}|E| \gg_{n}|A|^{\frac{1}{n+1}-O_{n}(\epsilon)}
$$


as required. Now Prop. 2.2 implies that that there are at most $\mid A_{k_{\dagger \dagger}}{ }^{\frac{1}{n+1}+O_{n}(\epsilon)} \ll_{n}$ $|A|^{\frac{1}{n+1}+O_{n}(\epsilon)}$ distinct tuples $\kappa(g)$, for $g$ a semisimple element in $A$. Indeed the same upper bound holds for tuples $\kappa\left(h_{0} g\right)$, for $g \in A$ such that $h_{0} g$ is simple. Prop. 3.4 implies, therefore, that there are $\gg_{n}|A|^{1-\frac{1}{n+1}-O(\epsilon)}$ elements in $\mathrm{Cl}_{i, t}^{\prime}(A)$ as required.

The final statement of the corollary follows immediately from Prop. 3.4.

\section{Moving From CONJUGaCy Classes to tRACES}

Cor. 3.5 gives us a bound on the size of the set $\mathrm{Cl}_{i, t}^{\prime}\left(A_{l}\right)$. In order to prove that small sets grow we will need to work with the tuple $\operatorname{Tr}_{i, t}^{\prime}\left(A_{l}\right)$. To make the transfer from conjugacy classes to traces we introduce the concept of wealth. Fix $l_{\dagger}$, the value for $l$ given by Cor. 3.5, $t$ a regular semisimple element; and $i$ an element of $\{0, \ldots, n\}$. The wealth of an element $r$ of $K$ is defined to be

$$
\diamond_{t, i}(r)=\mid\left\{\operatorname{cl}(g): \operatorname{tr}\left(t^{i} g\right)=r, g \in A_{l_{\dagger}} \text { and } t^{i} g \text { is semisimple }\right\} \mid .
$$

Now fix $\epsilon$ a small positive number; for $t \in A_{k_{\dagger}} \cap T(K)$ we define $A_{t,\left(j_{0}, j_{1}, \ldots, j_{n}\right)}$ where $\left(j_{0}, \ldots, j_{n}\right)$ is a tuple of integers:

$$
\begin{aligned}
A_{t,\left(j_{0}, j_{1}, \ldots, j_{n}\right)}=\left\{g \in A_{l_{+}} \mid\right. & 2^{j_{0}}<\diamond_{t, 0}(\operatorname{tr}(g)) \leq 2^{j_{0}+1} \\
& 2^{j_{1}}<\diamond_{t, 1}(\operatorname{tr}(t g)) \leq 2^{j_{1}+1}, \ldots, \\
& 2^{j_{n}}<\diamond_{t, n}\left(\operatorname{tr}\left(t^{n} g\right)\right) \leq 2^{j_{n}+1}, \text { and } \\
& \left.g, t g, \ldots, t^{n} g \text { are semisimple }\right\} .
\end{aligned}
$$

Thus $A_{t,\left(j_{0}, j_{1}, \ldots, j_{n}\right)}$ is a set of elements in $A_{l_{\dagger}}$ such that the corresponding tuple of traces of each element represents a roughly constant number of conjugacy classes. We often write $A_{t, j}$ for $A_{t,\left(j_{0}, \ldots, j_{n}\right)}$.

Note that this definition is dependent on our generating set $A$, on the constant $\epsilon$, and on the element $t$; note, furthermore, that there are at most $\left(\log _{2}|A|\right)^{n+1}$ tuples $\left(j_{0}, \ldots, j_{n}\right)$ such that $A_{t,\left(j_{0}, j_{1}, \ldots, j_{n}\right)}$ is non-empty.

Lemma 4.1. Suppose that $T$ is a rich torus and take $t \in T(K)$. Suppose that $\left|A_{t, \vec{j}}\right| \geq$ $|A|^{1-O(\epsilon)}$. Then

$$
2^{\max _{i} j_{i}-\min _{i} j_{i}} \ll d|A|^{O(\epsilon)}
$$

provided that $t$ lies outside a proper subvariety $W \subset T$ of degree $\ll_{n} 1$.

Note that the subvariety $W$ will be defined so as to include all of the non-regular elements.

Proof. By Cor. 3.5, the number of different tuples $\operatorname{Tr}_{\widehat{i}, t}(g)$, as $g$ varies over $A_{t, \vec{j}}$, is at least

$$
\frac{\mathrm{Cl}_{i, t}^{\prime}(A)}{2^{j_{0}+\cdots+j_{i-1}+j_{i+1}+\cdots+j_{n}+n}} \gg_{n} \frac{|A|^{\frac{n}{n+1}}-O(\epsilon)}{2^{j_{0}+\cdots+j_{i-1}+j_{i+1}+\cdots+j_{n}+n}} .
$$

On the other hand

$$
\left|\left\{\operatorname{tr}\left(t^{i} g\right) \mid g \in A_{t, \vec{j}}, 2^{j_{i}}<\diamond_{t, i}\left(\operatorname{tr}\left(t^{i} g\right)\right) \leq 2^{j_{i}+1}\right\}\right| \leq \frac{\left|\operatorname{cl}\left(A_{i+l_{\dagger}}^{\prime}\right)\right|}{2^{j_{i}}} .
$$


So, by Prop. 2.2 the number of different tuples $\operatorname{Tr}_{\widehat{i}, t}(g)$ can take on is at most

$$
\frac{\left|\operatorname{cl}\left(A_{n+l_{\dagger}}^{\prime}\right)\right|^{n}}{2^{j_{0}+\cdots+j_{i-1}+j_{i+1}+\cdots+j_{n}}} \ll_{n} \frac{|A|^{\frac{n}{n+1}}+O_{n}(\epsilon)}{2^{j_{0}+\cdots+j_{i-1}+j_{i+1}+\cdots+j_{n}}} .
$$

Now we need a definition:

$$
\operatorname{Tr}_{\widehat{i}, \widehat{k}, t}(g)=\left(\operatorname{tr}(g), \operatorname{tr}(t g), \ldots, \widehat{\operatorname{tr}\left(t^{i} g\right)}, \ldots, \widehat{\operatorname{tr}\left(t^{k} g\right)}, \ldots, \operatorname{tr}\left(t^{n} g\right)\right) .
$$

Again the number of different tuples $\operatorname{Tr}_{\hat{i}, \widehat{k}, t}(g)$ as $g$ varies over $A_{t, \vec{j}}$ is at most

$$
\frac{\left|\operatorname{cl}\left(A_{n k+l_{\dagger}}^{\prime}\right)\right|^{n-1}}{2^{j_{0}+\cdots+j_{i-1}+j_{i+1}+\cdots+j_{k-1}+j_{k+1}+\cdots+j_{n}}} \ll_{n} \frac{|A|^{\frac{n-1}{n+1}+O_{n}(\epsilon)}}{2^{j_{0}+\cdots+j_{i-1}+j_{i+1}+\cdots+j_{k-1}+j_{k+1}+\cdots+j_{n}}} .
$$

Then, for a given tuple $\vec{r}=\left(r_{0}, \ldots, r_{i-1}, r_{i+1}, \ldots, r_{k-1}, r_{k+1}, \ldots, r_{n}\right) \in K^{n-1}$ such that $r_{i}=\operatorname{tr}\left(t^{i} g\right)$ for some $g \in A_{t, \vec{j}}$, and a given index $k$, there are at least

$$
\begin{aligned}
& \gg_{n}\left(\frac{|A|^{\frac{n}{n+1}}-O(\epsilon)}{2^{j_{0}+\cdots+j_{i-1}+j_{i+1}+\cdots+j_{n}}}\right) \times\left(\frac{|A|^{\frac{n-1}{n+1}+O(\epsilon)}}{2^{j_{0}+\cdots+j_{i-1}+j_{i+1}+\cdots+j_{k-1}+j_{k+1}+\cdots+j_{n}}}\right)^{-1} \\
& =\frac{|A|^{\frac{1}{n+1}}-O(\epsilon)}{2^{j_{k}}}
\end{aligned}
$$

values for $\operatorname{tr}\left(t^{k} g\right)$ such that $\operatorname{Tr}_{\hat{i}, t}(g)=\left(r_{0}, \ldots, r_{i-1}, r_{i+1}, \ldots, r_{k-1}, \operatorname{tr}\left(t^{k} g\right), r_{k+1}, \ldots, r_{n}\right)$ for some $g \in A_{l_{\dagger}}$. Now we wish to apply Lem. 3.2, to do this we must assume, as we do, that $t$ lies outside an exceptional subvariety $W$ of bounded degree. Then Lem. 3.2 implies that there exist $a_{1}, \ldots, a_{k-1}, a_{k+1}, \ldots, a_{n} \in K$ such that, for all $g \in G(K)$,

$$
\operatorname{tr}\left(t^{k} g\right)=a_{1} \operatorname{tr}(g)+\cdots a_{k-1} \operatorname{tr}\left(t^{k-1} g\right)+a_{k+1} \operatorname{tr}\left(t^{k+1} g\right)+\cdots a_{n} \operatorname{tr}\left(t^{n} g\right) .
$$

We conclude, therefore, that (4.1) is a lower bound on values for $\operatorname{tr}\left(t^{i} g\right)$ such that

$$
\operatorname{Tr}_{\widehat{k}, t}(g)=\left(r_{1}, \ldots, r_{i-1}, \operatorname{tr}\left(t^{i} g\right), r_{i+1}, \ldots, r_{k-1}, r_{k+1}, \ldots, r_{n+1}\right) .
$$

Observe that there are at least $2^{j_{i}}$ values of $\operatorname{cl}\left(t^{i} g\right)$ for a fixed value of $\operatorname{tr}\left(t^{i} g\right)$. Thus

$$
|\operatorname{cl}(A)| \geq 2^{j_{i}} \frac{|A|^{\frac{1}{n+1}}-O(\epsilon)}{2^{j_{k}}} .
$$

Since $|\operatorname{cl}(A)| \ll|A|^{\frac{1}{n+1}+O(\epsilon)}$ we conclude that $2^{j_{i}-j_{k}} \ll|A|^{O(\epsilon)}$ as required.

\section{SMall SETS GROW}

We continue to analyse our set $A$ that "doesn't grow". Let $T$ be a rich torus; by the pigeonhole principle, every $t \in T(K)$ has a "popular tuple", $\vec{j}$, such that $\left|A_{t, \vec{j}}\right| \geq$ $|A|^{1-O(\epsilon)}$. In this case Lem. 4.1 implies that $\max _{i} j_{i}-\min _{i} j_{i} \ll O(\epsilon) \log _{2}|A|$. Define an integer

$$
p \rightarrow \vec{j}=\mid\left\{t \in T(K) \mid t \notin W(K) \text { and }\left|A_{t, \vec{j}}\right| \geq|A|^{1-O(\epsilon)}\right\} \mid
$$

where $W$ is as in Lem. 4.1,

Lem. 3.2 implies that, for $t \in T(K) \backslash W(K)$, the linear forms

$$
l_{i}: G(K) \rightarrow K, g \mapsto \operatorname{tr}\left(t^{i} g\right)
$$


are linearly dependent; what is more any subset of $n$ of these is linearly independent. These facts allow us to define

$$
f: T(K) \backslash W(K) \rightarrow K^{n}, t \mapsto \vec{r}=\left(r_{0}, \ldots, r_{n-1}\right)
$$

where $\vec{r}$ satisfies the equation

$$
\operatorname{tr}\left(t^{n} g\right)=r_{0} \operatorname{tr}(g)+r_{1} \operatorname{tr}(t g)+\cdots+r_{n-1} \operatorname{tr}\left(t^{n-1} g\right)
$$

for all $g \in G$.

We need to know that $f$ is, in some sense, close to being one-to-one. The following lemma gives the result that we need 3

Lemma 5.1. Suppose that $S$ is a subset of $T(\bar{K}) \backslash W(\bar{K})$, where $W$ is as above. Then

$$
|f(S)| \geq \frac{1}{n !}|S| \text {. }
$$

Proof. Recall the definition of $\kappa(t)=\left(a_{1}, \ldots, a_{n-1}\right)$ given at the end of Section 2. We apply the Cayley-Hamilton theorem (which states that a square matrix $t$ satisfies the polynomial $\operatorname{det}(\lambda I-t)=0)$ to conclude that

$$
\begin{aligned}
& \operatorname{det}(\lambda I-t)=\lambda^{n}+a_{n-1} \lambda^{n-1}+\cdots+a_{1} \lambda+(-1)^{n} ; \\
\Rightarrow & t^{n}=-a_{n-1} t^{n-1}-a_{n-2} t^{n-2}-\cdots-a_{1} t-(-1)^{n} ; \\
\Rightarrow & t^{n} g=-a_{n-1} t^{n-1} g-a_{n-2} t^{n-2} g-\cdots-a_{1} t g-(-1)^{n} g \text { for all } g \in G(K) ; \\
\Rightarrow & \operatorname{tr}\left(t^{n} g\right)=-a_{n-1} \operatorname{tr}\left(t^{n-1} g\right)-\cdots-a_{1} \operatorname{tr}(t g)-(-1)^{n} \operatorname{tr}(g) \text { for all } g \in G(K) ; \\
\Rightarrow & r_{n-1}=-a_{n-1}, r_{n-2}=-a_{n-2}, \ldots, r_{1}=-a_{1}, r_{0}=(-1)^{n+1} .
\end{aligned}
$$

Now, all elements in $T(\bar{K}) \backslash W(\bar{K})$ are regular; write $T(\bar{K})$ as the set of diagonal matrices in $G(\bar{K})$. Then two elements $g, h \in T(\bar{K}) \backslash W(\bar{K})$ satisfy $\kappa(g)=\kappa(h)$ if and only if there is an element $\sigma$ in the symmetric group on $n$ letters such that $g_{i i}=h_{\sigma(i) \sigma(i)}$. The result follows.

Now set $\overrightarrow{j^{\mathrm{max}}}=\left(j_{0}^{\max }, \ldots, j_{n}^{\max }\right)$ be the tuple for which $p \rightarrow$ is maximal (so $\overrightarrow{j^{\mathrm{max}}}$ is the "most popular" popular tuple). Define

$$
D=\left\{t \in T(K) \cap A_{k_{\dagger}} \mid t \notin V(K) \cup W(K) \text { and }\left|A_{t, \overrightarrow{j^{\max }}}\right| \geq|A|^{1-O(\epsilon)}\right\} .
$$

Observe that $|D|=p_{j_{\text {max }}}$. Since there are at most $\left(\log _{2}|A|\right)^{n}$ choices for $\overrightarrow{j^{\mathrm{max}}}$, Lem. 2.6 implies that $|D| \gg_{n}|A|^{\frac{1}{n+1}}-O(\epsilon)$.

We also define $j_{\max }^{\max }\left(\right.$ resp. $\left.j_{\min }^{\max }\right)$ to be the maximum (resp. minimum) element in $\left\{j_{0}^{\max }, \ldots, j_{n}^{\max }\right\}$.

We need the following result from arithmetic combinatorics; it is a strengthening of [He2, Cor. 3.8].

Proposition 5.2. There exists $c>0$ such that, for any $n>2$ a positive integer and any $\delta>0$, there exist $e, \eta>0$ such that

- for every prime $p$, and field $K=\mathbb{Z} / p \mathbb{Z}$;

\footnotetext{
${ }^{3}$ We thank an anonymous referee for significantly simplifying the proof of this result.
} 
- for every set $X \subset K$ such that $|X| \leq p^{1-\delta}$;

- for every set $Y \subset K^{n}$;

- for each $\vec{y} \in Y$, and $X_{\vec{y}} \subseteq X^{n}$ such that

$$
\vec{y} \cdot X_{\vec{y}}=\left\{y_{1} x_{1}+\cdots+y_{n} x_{n}: \vec{x} \in X_{\vec{y}}\right\}
$$

is contained in $X$;

either $|Y|<|X|^{\text {cn }}$ or

$$
\left|X_{\vec{y}}\right| \leq e|X|^{n-\eta} \text { for some } \vec{y} \in Y .
$$

Proof. Suppose $|Y| \geq|X|^{c n \eta}$ (c>0 to be chosen later) and $\left|X_{\vec{y}}\right| \gg|X|^{n-\eta}$ for every $\vec{y} \in Y$. We will try to derive a contradiction.

There is a coordinate $1 \leq i \leq n$ such that the image $\pi_{i}(Y)$ of $Y$ under the projection $\pi_{i}$ to the $i$ th coordinate has $\geq|Y|^{1 / n}$ elements. We can assume without loss of generality that $n=1$. Let $Y^{\prime} \subset Y$ be a maximal set such that $\left.\pi_{1}\right|_{Y^{\prime}}$ is injective. Then $\left|Y^{\prime}\right| \gg|Y|^{1 / n}$.

For $x_{3}, x_{4}, \ldots, x_{n} \in X$, let

$$
R_{x_{3}, x_{4}, \ldots, x_{n}}=\left\{\left(\vec{y}, x_{1}, x_{2}\right) \in Y^{\prime} \times X \times X:\left(x_{1}, x_{2}, \ldots, x_{n}\right) \in X_{\vec{y}}\right\} .
$$

By assumption, $\sum_{x_{3}, \ldots, x_{n} \in X}\left|R_{x_{3}, \ldots, x_{n}}\right| \gg\left|Y^{\prime}\right||X|^{n-\eta}$, and so we can fix $x_{3}, x_{4}, \ldots, x_{n} \in X$ such that $S=R_{x_{3}, \ldots, x_{n}}$ has $\gg\left|Y^{\prime}\right||X|^{2-\eta}$ elements. For $\vec{y} \in Y^{\prime}$, let $c_{\vec{y}}=x_{3} y_{3}+\ldots+x_{n} y_{n}$.

We have $S \subset Y^{\prime} \times X \times X$; let $S_{\vec{y}}=\left\{\left(x_{1}, x_{2}\right) \in X \times X:\left(\vec{y}, x_{1}, x_{2}\right) \in S\right\}$. There is an $\epsilon>0$ such that the sets $S_{\vec{y}}$ with $\left|S_{\vec{y}}\right| \leq \epsilon|X|^{2-\eta}$ contribute at most $\frac{1}{2}|S|$ elements to $S$. Let $Y^{\prime \prime}$ be the set of all $\vec{y} \in Y^{\prime}$ with $\left|S_{\vec{y}}\right|>\epsilon|X|^{2-\eta}$. Then $\sum_{\vec{y} \in Y}\left|S_{\vec{y}}\right| \geq \frac{1}{2}|S| \gg\left|Y^{\prime}\right||X|^{2-\eta}$ and so $\left|Y^{\prime \prime}\right| \gg\left|Y^{\prime}\right||X|^{\eta} \gg|X|^{(c-1) \eta}$. By the definition of $S_{\vec{y}}$,

$$
\left\{x_{1} y_{1}+x_{2} y_{2}+c_{\vec{y}}:\left(x_{1}, x_{2}\right) \in S_{\vec{y}}\right\} \subset X .
$$

Thus, for all $\vec{y} \in Y^{\prime \prime}$, there are $>\epsilon|X|^{2-\eta}$ distinct pairs $\left(x, x^{\prime}\right)=\left(x_{1}, x_{1} y_{1}+x_{2} y_{2}+c_{\vec{y}}\right) \epsilon$ $X \times X$ such that $x^{\prime}-y_{1} x \in y_{2} X+c_{\vec{y}}$. Thus the energy $E_{+}\left(X, y_{1} X\right)=\sum_{d \in X-y_{1} X} \mid\{a \in$ $\left.X, b \in y_{1} X: a-b=d\right\}\left.\right|^{2}$ is greater than $\frac{1}{\left|y_{2} X+c_{\vec{y}}\right|}\left(\epsilon|X|^{2-\eta}\right)^{2}=\epsilon^{2}|X|^{3-2 \eta}$. Hence

$$
\sum_{y_{1} \in \pi\left(Y^{\prime \prime}\right)} E\left(X, y_{1} X\right)>\epsilon^{2} \pi_{1}\left(Y^{\prime \prime}\right)|X|^{3-2 \eta}
$$

Recall that $\left.\pi_{1}\right|_{Y^{\prime}}$ is injective and $Y^{\prime \prime} \subset Y^{\prime}$ has $\gg|X|^{(c-1) \eta}$ elements; thus $\left|\pi_{1}\left(Y^{\prime \prime}\right)\right| \gg$ $|X|^{(c-1) \eta}$.

Hence, for $c$ and $p$ large enough and $\eta$ smaller than a constant times $\delta$, (5.2) is contradiction with Theorem $\mathrm{C}$ of $\mathrm{BO}$. (If $p$ is smaller than a constant, the statement we are trying to prove is trivially true.)

We are now able to prove our main result.

Main Theorem. Let $G=\mathrm{SL}_{n}$ and fix $\delta$ a positive number. Then there are positive numbers $\epsilon$ and $C$ such that, for all fields $K=\mathbb{Z} / p \mathbb{Z}$ (p prime), and all sets $A \subset G(K)$ that generate $G(K)$, either $|A|<p^{n+1-\delta}, \delta>0$ or $|A \cdot A \cdot A| \geq C|A|^{1+\epsilon}$. 
Proof. Suppose that $|A|<p^{n+1-\delta}$ and that $A$ does not grow; we seek a contradiction to Prop. 5.2. Let $\eta$ be some (small) positive number.

First set $R=K=\mathbb{Z} / p \mathbb{Z}$. We define $X$ to be the following set:

$$
\left\{\operatorname{tr}\left(t^{i} a\right) \mid t \in D, a \in A_{t, \overrightarrow{j^{\mathrm{max}}}}, i=0, \ldots, n ; \text { and } t^{i} a \text { is semisimple }\right\} .
$$

Observe that $|X| \leq \frac{\left|\operatorname{cl}\left(\left(A_{n k_{\dagger}+l_{\dagger}}\right)^{\prime}\right)\right|}{2^{j_{\min }^{\max }}}$ where $\left(A_{n k_{\dagger}+l_{\dagger}}\right)^{\prime}$ is the set of semisimple elements in $A_{n k_{\dagger}+l_{\dagger}}$. Prop. 2.2 implies that $\left|\operatorname{cl}\left(A_{n k_{\dagger}+l_{\dagger}}\right)^{\prime}\right| \ll_{n}|A|^{\frac{1}{n+1}+O(\epsilon)}$, and so

$$
|X| \leq \frac{|A|^{\frac{1}{n+1}}+O(\epsilon)}{2^{j_{\min }^{\max }}} .
$$

Thus if we pick $\epsilon$ small enough we know that $|X|<p^{1-\delta^{\prime}}$ for some positive number $\delta^{\prime}$, and we have satisfied the assumption in Prop. 5.2,

Now define $Y=f(D)$ and for $\vec{y} \in Y$ we define $X_{\vec{y}}$ to be the set of elements $\vec{x}$ in $X^{n}$ such that $\vec{y} \cdot \vec{x}$ is equal to the trace of a semisimple element in $D A$, i.e. such that $\vec{y} \cdot \vec{x}$ lies in $X$.

Our first job is to show that $\left|X_{\vec{y}}\right| \gg_{n}|X|^{n-\eta}$ for all $\vec{y} \in Y$. Take $\vec{y}=f(t)$ for some $t \in D$. Then $\vec{y}=\left(r_{0}, \ldots, r_{n-1}\right)$ where $\vec{r}$ satisfies the equation

$$
\operatorname{tr}\left(t^{n} g\right)=r_{0} \operatorname{tr}(g)+r_{1} \operatorname{tr}(t g)+\cdots+r_{n-1} \operatorname{tr}\left(t^{n-1} g\right)
$$

for all $g \in G$.

Now Prop. 3.4 implies that, for all $i=0, \ldots, n$,

$$
\left|\mathrm{Cl}_{\hat{i}, t}\left(A_{t, \overrightarrow{j^{\max }}}\right)\right| \gg_{n}\left|A_{t, \overrightarrow{j^{\max }}}\right|^{\frac{n}{n+1}+O(\epsilon)} \gg_{n}|A|^{\frac{n}{n+1}+O(\epsilon)} .
$$

Thus we conclude that

$$
\left|\operatorname{Tr}_{\hat{i}, t}\left(A_{t, \overrightarrow{j^{\max }}}\right)\right| \gg \frac{|A|^{\frac{n}{n+1}+O(\epsilon)}}{2^{j_{0}^{\max }+\cdots+j_{n}^{\max }+n}} .
$$

Next observe that Lem. 4.1 implies that the wealth for each entry in the tuple differs only by a factor of $O(\epsilon)$, hence we are able to conclude that

$$
\left|\operatorname{Tr}_{\hat{i}, t}(A)\right| \gg \frac{|A|^{\frac{n}{n+1}-O(\epsilon)}}{\left(2^{j_{\min }^{\max }}\right)^{n}} \gg|X|^{n-O(\epsilon)} .
$$

If we pick $\epsilon$ to be small enough in terms of $\eta$ then this implies that $\left|\operatorname{Tr}_{\hat{i}, t}(A)\right| \gg|X|^{n-\eta}$, as required.

Finally we need to show that $|Y| \gg|X|^{O(n \eta)}$. Now Lem. 5.1 implies that

$$
|Y|=|f(D)| \gg_{n}|A|^{\frac{1}{n+1}+O_{n}(\epsilon)} .
$$

We conclude that, provided we choose $\epsilon$ small enough with respect to $n$ and $\eta$, then $|Y| \gg_{n}|X|^{\eta}$. Thus we have a contradiction with Prop. 5.2 as required. 


\section{Generalisations}

We give a brief explanation as to how ideas from the current paper may be applied more generally. It should be a relatively straightforward matter to strengthen the Main Theorem to the following statement: for every $\delta>0$ there is an $\epsilon>0$ such that if $|A|<p^{n+1-\delta}$ or if $p^{n+1+\delta}<|A|<p^{2 n+2-\delta}$, then

$$
|A \cdot A \cdot A| \gg_{n}|A|^{1+\epsilon}
$$

where $\epsilon$ and the implied constant depend only on $n$.

To prove this statement, our current proof would need to be extended to consider vectors of the form

$$
\left(\begin{array}{c}
\operatorname{tr}(g) \\
\operatorname{tr}\left(g^{-1}\right)
\end{array}\right)
$$

for $g \in A$; analysis of the set of all such vectors would lead to a contradiction of a result similar to Prop. 5.2, but over the ring $K \times K$, rather than over $K$. This would be similar to the approach adopted in [He2, $\S 6]$, but would also need a concept of wealth for it to work in the current setting.

As it is we have restricted our attention simply to the set of traces, $\{\operatorname{tr}(g) \mid g \in A\}$. We did this because things are more transparent in this setting; but more importantly we did this because there seems no obvious way of extending the ideas in this paper to cover sets of size greater than $p^{2 n+2-\delta}$.

The significance of $\operatorname{tr}(g)$ and $\operatorname{tr}\left(g^{-1}\right)$ is that these are entries in the tuple $\kappa(g)$ (the first and last entry respectively). Both of these entries correspond to the fundamental $n$-dimensional representations of $G$ over $\bar{K}$; when $n=3$ these are all of the fundamental representations of $G$ and hence an analysis of these two representations gives information about sets of size all the way up to $|G|^{1-\delta}$.

When $n \geq 4$, however, the other entries in $\kappa$ correspond to representations of dimension strictly greater than $n$. This fact prevents us from using them as we use $\operatorname{tr}(g)$ and $\operatorname{tr}\left(g^{-1}\right)$.

The methods in the present paper seem to extend easily to $\mathrm{Sp}_{2 n}, n \geq 2$; there they show that $|A \cdot A \cdot A| \geq|A|^{1+\epsilon}$ for every $A \subset \operatorname{Sp}_{2 n}(K)$ with $|A|<p^{2 n+1-\delta}, \delta>0$. However, there is a technical reason why the extension of these methods to $\mathrm{SO}_{n}$ would be more problematic: both $\mathrm{SL}_{n}$ and $\operatorname{Sp}_{2 n}$ obey $(\operatorname{dim}(\pi)+1) \cdot \operatorname{dim}(T) \leq \operatorname{dim}(G)$, where $\pi$ is the smallest-dimensional faithful representation of $G$ and $T$ is the maximal torus of $G$. In contrast, in the case of $\mathrm{SO}_{n},(\operatorname{dim}(\pi)+1) \cdot \operatorname{dim}(T)=(2 m+1) \cdot m>\frac{n(n-1)}{2}=\operatorname{dim}(G)$ if $n=2 m$, and $(\operatorname{dim}(\pi)+1) \cdot \operatorname{dim}(T)=(2 m+2) \cdot m>\frac{n(n-1)}{2}=\operatorname{dim}(G)$ if $n=2 m+1$.

$$
* * *
$$

The difficulties in extending the result to $\mathrm{SO}_{n}$ complicate the following approach to proving that every subset $A \subset \mathrm{SL}_{n}(K)$ grows (as usual, $K=\mathbb{F}_{p}$ here). Let $A \subset \mathrm{SL}_{n}\left(\mathbb{F}_{p}\right)$, $|A| \geq p^{n-1+\delta}, \delta>0$ and consider the natural action of $S L_{n}(K)$ on $V=K^{n}$. Let $H$ be a maximal parabolic subgroup of $\mathrm{SL}_{n}\left(\mathbb{F}_{p}\right)$ stabilizing a one-dimensional subspace $\langle\vec{v}\rangle$ of $V$, i.e.,

$$
H=\left\{g \in \mathrm{SL}_{n}\left(\mathbb{F}_{p}\right): g \vec{v}=\lambda \vec{v} \text { for some } \lambda \in K^{*}\right\} .
$$

Then $[G: H] \ll p^{n-1}$, and so (by [He2, Lemma 7.2]), $\left|A^{-1} A \cap H\right| \gg p^{\delta}$. Then one can try to show that $A^{-1} A \cap H$ grows in $H$; from this, it would follow that $A$ itself grows $([\mathrm{He} 2$, 
Lemma 7.3]). The problem here is that we could have $\left\langle A^{-1} A \cap H\right\rangle \sim \mathrm{SO}_{n-1}$, for example. This possibility could be avoided if we could find a rich torus $T$ in $H$, or, conversely, if we could choose $H$ so that it contains a rich torus $T$.

The reason why this would be enough is the following. Suppose $\left\langle A^{-1} A \cap H\right\rangle \sim \mathrm{SO}_{n-1}$, or even $\left\langle A^{-1} A \cap H\right\rangle \sim K^{*} \times \mathrm{SO}_{n-1}$. The maximal torus of $K^{*} \times \mathrm{SO}^{n-1}$ has smaller dimension than that of $\mathrm{SL}_{n}$. Hence, since $A^{-1} A \cap T(K)$ is contained in $A^{-1} A \cap H \subset$ $\left\langle A^{-1} A \cap H\right\rangle \sim K^{*} \times \mathrm{SO}_{n-1}$, we cannot possibly have $\left|A_{k} \cap T(K)\right| \gg|A|^{\operatorname{dim}(T) / \operatorname{dim}(G)}$ (see Prop. 2.3 in the present paper), i.e., $T$ cannot be rich.

In general, by the same argument, if $T$ is rich and contained in $H$, then $A^{-1} A \cap H$ cannot be contained in any algebraic subgroup of $H$ of bounded degree whose maximal tori are of smaller dimension than $T$. The only connected algebraic subgroups $H^{\prime}$ of $H$ whose maximal tori are of the same dimension as a maximal torus of $\mathrm{SL}_{n}$ have form

$$
U \rtimes\left(\left(\mathrm{SL}_{m_{1}} \times \ldots \times \mathrm{SL}_{m_{k}}\right) T\right),
$$

where $U$ is a unipotent group, $m_{1}+m_{2}+\ldots+m_{k}=n$, and $T$ is a maximal torus.

It follows that $A^{-1} A \cap H$ is contained in one such group $H^{\prime}$, but not in any proper algebraic subgroup of $H^{\prime}$ of bounded degree. By [He2, Prop. 4.2], we conclude that $A^{-1} A \cap H$ is not contained in any algebraic subvariety of $H^{\prime}$ of bounded degree. Thus, we can work as if $A^{-1} A \cap H$ generated $H^{\prime}$. By induction on $n$, the theorem $|A \cdot A \cdot A| \geq|A|^{1+\epsilon}$ would then be proven for all $A \subset \mathrm{SL}_{n}\left(\mathbb{F}_{p}\right)$ of arbitrary size $|A| \leq\left|\mathrm{SL}_{n}\left(\mathbb{F}_{p}\right)\right|^{1-\delta}, \delta>0$.

The only gap in the above argument is that one must still prove that there exists a maximal parabolic subgroup $H$ containing a rich torus $T$. If a rich torus $T$ fixes some vector $\vec{v} \in K^{n}$, then $T$ lies in a maximal parabolic subgroup $H_{\vec{v}}$ of the required type. The only problem remaining, then, is to find a rich torus $T$ (i.e., a torus $T$ with $\left|A_{k} \cap T(K)\right| \gg$ $\left.|A|^{\frac{\operatorname{dim} T}{\operatorname{dim} G}-O(\epsilon)}\right)$ such that $T$ has at least one eigenvector in $K^{n}$ (as opposed to just in $\bar{K}^{n}$ ).

One possible approach to this last question is to look for regular semisimple elements in $A^{-1} A \cap H$, where $H$ is a maximal parabolic subgroup. If there is one such element, there will be many, and hence there will be a rich maximal torus $T$ inside $H$. If instead there are many non-semisimple elements, one can conjugate them by coset representatives of $H$ to produce $\gg|A|$ non-semisimple elements of $A_{k}$; this, in turn, results in $|A \cdot A \cdot A| \geq|A|^{1+\epsilon}$ by an argument sticking varieties in different directions (as in [He2, §4.3]). The only problem is that $A^{-1} A \cap H$ may consist almost entirely of non-regular semisimple elements, as then too many of their conjugates could be identical; a different argument would be needed in this case.

6.1. Final note. Shortly after the completion of this paper, and well after the completion of the research leading to it, Pyber and Szabo [PS], and Breuillard, Green, and Tao [BGT] independently announced that they had proven results more general than those in the present paper. According to these announcements, their methods are based in part on those of the second author [He1, He2], on which the present work also rests.

Funding. This work was supported by EPSRC (Engineering and Physical Sciences Research Council) grant EP/E054919/1.

Acknowledgements. We would like to thank R. Hill, M. Kassabov and N. Nikolov for interesting conversations on subgroups and tori in $\mathrm{SL}_{n}$. In addition, K. Raghavan, A. 
Zalesski and S. Murray made useful comments. We are likewise grateful for the hospitality of the people of Orsay (Paris Sud XI).

\section{REFERENCES}

[Bo] Bourgain, J. "Multilinear exponential sums in prime fields under optimal entropy condition on the sources." Geom. funct. anal. 18 (2009): 1477-1502.

[BG] Bourgain, J., and A. Gamburd. "Uniform expansion bounds for Cayley graphs of $\mathrm{SL}_{2}\left(\mathbb{F}_{p}\right)$. " Ann. of Math. 167 no. 2 (2008): 625-642.

[BGS] Bourgain, J., Gamburd, A., and P. Sarnak. "Affine linear sieve, expanders, and sum-product." Invent. Math., to appear.

[BGT] Breuillard, E., Green, B., and T. Tao. "Linear approximate groups." Preprint: arxiv.org:1001.4570.

[Di] Dinai, O. "Expansion properties of finite simple groups." Preprint.

[He1] Helfgott, H. A. "Growth and generation in $\mathrm{SL}_{2}(\mathbb{Z} / p \mathbb{Z})$." Ann. of Math. (2) 167, no. 2 (2008): 601-623.

[He2] Helfgott, H. A. "Growth in $\mathrm{SL}_{3}(\mathbb{Z} / p \mathbb{Z}) . "$ To appear in J. Eur. Math. Soc., preprint available at: arxiv.org:0807.2027.

[MCC] Chang, M.-C. "Product theorems in $\mathrm{SL}_{2}$ and $\mathrm{SL}_{3} . "$ J. Math. Jussieu. 7 (2008): 1-25.

[PS] Pyber, L., and E. Szabó. "Growth in finite simple groups of Lie type." Preprint available at: arxiv.org:1001.4556.

[V] Varjú, P., "Expansion in $\mathrm{SL}_{d}\left(\mathscr{O}_{K} / I\right), I$ squarefree." Preprint.

School of Mathematics, University of Bristol, Bristol, BS8 1TW, United Kingdom 


\title{
GROWTH OF SMALL GENERATING SETS IN $\mathrm{SL}_{n}(\mathbb{Z} / p \mathbb{Z})$
}

\author{
NICK GILL AND HARALD ANDRÉS HELFGOTT
}

\begin{abstract}
Let $G=\mathrm{SL}_{n}$ and fix $\delta$ a positive number. We prove that there are positive numbers $\epsilon$ and $C$ such that, for all fields $K=\mathbb{Z} / p \mathbb{Z}$ ( $p$ prime), and all sets $A \subset G(K)$ that generate $G(K)$, either $|A|<p^{n+1-\delta}, \delta>0$ or $|A \cdot A \cdot A| \geq C|A|^{1+\epsilon}$.
\end{abstract}

In $\left[\mathrm{He} 1\right.$ and $\mathrm{He} 2$, the second author proved that every set of generators of $\mathrm{SL}_{2}\left(\mathbb{F}_{p}\right)$ and $\mathrm{SL}_{3}\left(\mathbb{F}_{p}\right)$ grows rapidly. This has since found numerous applications $\left.([\mathrm{BG}],[\mathrm{BGS}], \mathrm{V}]\right)$ and generalisations to groups of the same type $\left(\mathrm{SL}_{2}, \mathrm{SL}_{3}\right)$ over other fields ([MCC], Di]). In the present paper, we prove that small subsets of $\mathrm{SL}_{n}\left(\mathbb{F}_{p}\right)$ grow.

Main Theorem. Let $G=\mathrm{SL}_{n}$ and fix $\delta$ a positive number. Then there are positive numbers $\epsilon$ and $C$ such that, for all fields $K=\mathbb{Z} / p \mathbb{Z}$ ( $p$ prime), and all sets $A \subset G(K)$ that generate $G(K)$, either $|A|<p^{n+1-\delta}, \delta>0$ or $|A \cdot A \cdot A| \geq C|A|^{1+\epsilon}$.

Here $|S|$ denotes the number of elements of a finite set $S$.

Our basic approach is to assume that $A$ is a set of generators for $G(K)$ that does not grow; i.e., for a fixed positive $\epsilon,|A \cdot A \cdot A| \ll_{n}|A|^{1+\epsilon}$. We will then show that if $A$ is "small" $\left(|A|<p^{n+1-\delta}\right)$ we have a contradiction with a result from arithmetic combinatorics (Prop. 5.2) related to the sum-product theorem and to incidence theorems.

\section{Notation AND BACKGROUnd}

We collect here the background ideas that will be needed in the sequel.

1.1. Arithmetic combinatorics. Our notation in this area is standard and, in particular, is identical to that of [He2]. In this section $G$ is an arbitrary group.

Given a positive integer $r$ and a subset $S$ of a group $G$, we define

$$
A_{r}=\left\{g_{1} \cdot g_{2} \cdots g_{r} \mid g_{i} \in A \cup A^{-1} \cup\{1\}\right\} .
$$

Given real numbers $a, b, x_{1}, \ldots, x_{n}$, we write

$$
a \ll_{x_{1}, \ldots, x_{n}} b
$$

to mean that the absolute value of $a$ is at most the real number $b$ multiplied by a constant $c$ depending only on $x_{1}, \ldots, x_{n}$. In this situation we also write $a=O_{x_{1}, \ldots, x_{n}}(b)$. When we omit $x_{1}, \ldots, x_{n}$, and write $a \ll b$, we mean that the constant $c$ is absolute, unless we explicitly state otherwise.

Occasionally we may write vectors in place of $x_{1}, \ldots, x_{n}$; for instance $a \ll \vec{v} b$ where $a$ and $b$ are real and $\vec{v}=\left(v_{1}, \ldots, v_{n}\right) \in \mathbb{R}^{n}$. In this case we mean that $a$ is at most the real number $b$ multiplied by a constant $c$ depending only on $v_{1}, \ldots, v_{n}$, and on $n$.

The next result will be useful in its own right; it also gives some more context to the statement of the Main Theorem. 
Lemma 1.1. [He2, Lem. 2.2] (Tripling Lemma). Let $k>2$ be an integer and let $c$ and $\epsilon$ be positive numbers. Then there exist positive numbers $c^{\prime}$ and $\epsilon^{\prime}$ such that, if $A$ is a finite subset of a group $G$ satisfying $\left|A_{k}\right| \geq c|A|^{1+\epsilon}$, then

$$
|A \cdot A \cdot A| \geq c^{\prime}|A|^{1+\epsilon^{\prime}}
$$

We include one final piece of notation: For an $n$-tuple $\left(x_{1}, \ldots, x_{n}\right)$, where $x_{1}, \ldots, x_{n}$ are elements of some set, we write $\left(x_{1}, \ldots, \widehat{x_{i}}, \ldots, x_{n}\right)$ to mean the $n-1$-tuple

$$
\left(x_{1}, \ldots, x_{i-1}, x_{i+1}, \ldots, x_{n}\right) \text {. }
$$

1.2. Degree and Escape. We will use the notion of escape from subvarieties in a very similar way to in [He2]. A full explanation of this idea is given in [He2]; we note here only some essential definitions and results.

For $V$ an affine algebraic variety, we define $\operatorname{dim} V$ to be the dimension of the irreducible subvariety of $V$ of largest dimension. As for the degree, it will be best to see it as a vector: we define the degree $\overrightarrow{\operatorname{deg}}(V)$ of an arbitrary variety $V$ to be

$$
\left(d_{0}, d_{1}, \ldots, d_{k}\right)
$$

where $k=\operatorname{dim}(V)$ and $d_{j}$ is the degree of the union of the irreducible components of $V$ of dimension $j$.

If a regular map $\phi: V \mapsto W$ between two varieties $V \subset \mathbb{A}^{m}, W \subset \mathbb{A}^{n}$ is defined by polynomials $\phi_{1}, \phi_{2}, \ldots, \phi_{n}$ on the variables $x_{1}, x_{2}, \ldots, x_{m}$, we define $\operatorname{deg}_{p o l}(\phi)$ to be $\max _{j} \operatorname{deg}\left(\phi_{j}\right)$. (If several representations of $\phi$ by polynomials $\phi_{1}, \phi_{2}, \ldots, \phi_{n}$ are possible, we choose - for the purposes of defining $\operatorname{deg}_{p o l}$ - the one that gives us the least value of $\operatorname{deg}_{p o l}$.)

Before proceeding we note an abuse of language: for a variety $V$ defined over a field $K$, and a subvariety $W$ defined over the algebraic completion $\bar{K}$ of $K$, we will write $W(K)$ for $W(\bar{K}) \cap V(K)$.

The following series of results is related to the idea of escape and non-singularity.

Proposition 1.2. [He2, Prop. 4.1] Let $G$ be a group. Consider a linear representation of $G$ on a vector space $\mathbb{A}^{n}(K)$ over a field $K$. Let $V$ be an affine subvariety of $\mathbb{A}^{n}$.

Let $A$ be a subset of $G$; let $\mathscr{O}$ be an $\langle A\rangle$-orbit in $\mathbb{A}^{n}(K)$ not contained in $V$. Then there are constants $\eta>0$ and $m$ depending only on $\overrightarrow{\operatorname{deg}}(V)$ such that, for every $x \in \mathscr{O}$, there are at least $\max (1, \eta|A|)$ elements $g \in A_{m}$ such that $g x \notin V$.

Lemma 1.3. [He2, Lem 4.3] Let $X \subseteq \mathbb{A}^{m_{1}}$ and $Y \subseteq \mathbb{A}^{m_{2}}$ be affine varieties defined over a field $K$. let $f: X \rightarrow Y$ be a regular map. Let $V$ be a subvariety of $X$ such that the derivative $\left.D f\right|_{x=x_{0}}$ of $f$ at $x=x_{0}$ is a nonsingular linear map for all $x_{0}$ on $X$ outside $V$.

Then, for any $S \subseteq X(\bar{K}) \backslash V(\bar{K})$, we have

$$
|f(S)| \gg \underset{\operatorname{deg}(X), \operatorname{deg}_{\mathrm{pol}}(f)}{ }|S| .
$$

Lemma 1.4. [He2, Lem.'s 4.4 and 4.6] Let $n$ be a positive integer, $\left(a_{1}, \ldots, a_{d}\right)$ and $\left(b_{1}, \ldots, b_{e}\right)$ be tuples of non-negative integers. There exist $c, d>0$ and a positive integer $k$ such that

- for all fields $K$ such that $|K|>c$;

- for all reductive algebraic groups $G \subset \mathrm{GL}_{n}$ defined over $K$ of degree $\left(a_{1}, \ldots, a_{e}\right)$; 
- for all subvarieties $V$ of $G$ of degree $\left(b_{1}, \ldots, b_{e}\right)$;

- for all $A \subset G(K)$ which generate $G(K)$;

we have

$$
\left|A_{k} \cap(G(K) \backslash V(K))\right| \geq d|A| .
$$

Corollary 1.5. [He2, Cor. 4.5] Let $G \subset \mathrm{GL}_{n}$ be an algebraic group of rank $r$ and $Y \subset \mathbb{A}^{m}$ an affine variety, both defined over a field $K$. Let $f: G \rightarrow Y$ be a regular map. Let $V$ be a proper subvariety of $G$. Assume that the derivative $\left.D f\right|_{x}$ of $f$ at $x$ is a nonsingular linear map for all $x$ on $G$ outside $V$.

Let $A \subset G(K)$ be a set of generators of $G(K)$. Then

$$
\left|f\left(A_{k} \cap(G(K) \backslash V(K))\right)\right| \gg \overrightarrow{\operatorname{deg}}(G), \overrightarrow{\operatorname{deg}}(V), \operatorname{deg}_{\mathrm{pol}}(f)|A|,
$$

where $k \ll \overrightarrow{\operatorname{deg}(V)} 1$.

Lemma 1.6. [He2, Lem. 4.7] Let $G \subseteq G L_{n}$ be an algebraic group defined over a field $K$. Let $X / K$ and $Y$ be a affine varieties such that $\operatorname{dim}(Y)=\operatorname{dim}(G)$. Let $f: X \times G \rightarrow Y$ be a regular map. Define

$$
f_{x}: G \rightarrow Y, g \mapsto f(x, g) .
$$

Then there is a subvariety $Z_{T \times G} \subset T \times G$ such that, for all $\left(x, g_{0}\right) \in(X \times G)(\bar{K})$, the derivative

$$
\left.\left(D f_{x}\right)\right|_{g=g_{0}}:\left.\left.(T G)\right|_{g=g_{0}} \rightarrow(T Y)\right|_{f\left(x, g_{0}\right)}
$$

is non-singular if and only if $\left(x, g_{0}\right)$ does not lie on $Z_{T \times G}$. Moreover,

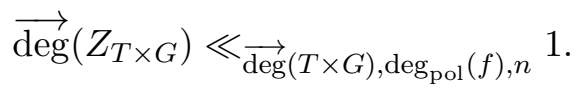

\section{KNOWN BOUNDS FOR SETS THAT DO NOT GROW.}

We set $G=S L_{n}, K=\mathbb{Z} / p \mathbb{Z}$; we use a number of results about generating sets in $G(K)$ that "do not grow" He2. We start with information about how such a set intersects with a torus of $G$.

Proposition 2.1. [He2, Cor.'s 5.4 and 5.10] Let $G=S L_{n}$.

(a) There exists $C>0$ and $k$ a positive integer such that, for all finite fields $K$ of characteristic $\neq 2$, for any $T$ a maximal torus of $G$ defined over $\bar{K}$, and for any $A \subset G(K)$ a set of generators of $G(K)$, we have $|A \cap T(K)| \leq C\left|A_{k}\right|^{\frac{1}{n+1}}$.

(b) There exist $d^{\prime}, \delta>0$ and $k^{\prime}$ a positive integer such that, for all finite fields $K$ of characteristic $\neq 2$, and for any $A \subset G(K)$ a set of generators of $G(K)$ satisfying $|A \cdot A \cdot A| \leq d|A|^{1+\epsilon}$ for some $d, \epsilon>0$, there is a maximal torus $T$ such that $\left|A_{k^{\prime}} \cap T(K)\right| \geq\left(d^{\prime} d^{\delta}\right)|A|^{\frac{1}{n+1}-\delta \epsilon}$.

A torus $T$ in $G$ that satisfies the last inequality will be called rich; that is to say, such a $T$ has a large intersection with the set $A_{k}$.

For a set $B \subset G(K)$ define $\operatorname{cl}(B)$ to be the set of conjugacy classes of $G(K)$ that intersect $B$; if $B$ contains only one element, $g$, then write $\operatorname{cl}(g)$ for $\operatorname{cl}(B)$. We also write $B^{\prime}$ for the set of semisimple elements in $B$; there are well-known bounds on $\left|\operatorname{cl}\left(A^{\prime}\right)\right|$ where $A$ is a generating set in $G(K)$ that does not grow. 
Proposition 2.2. [He2, Cor.'s 5.7 and 5.11] Let $G=S L_{n}$.

(a) There exists $C>0$ and a positive integer $k$ such that, for all finite fields $K$ of characteristic $\neq 2$, for any $T$ a maximal torus of $G$ defined over $\bar{K}$, and for any $A \subset G(K)$ a set of generators of $G(K)$, we have $\left|\operatorname{cl}\left(\left(A_{k}\right)^{\prime}\right)\right| \geq C|A|^{\frac{1}{n+1}}$.

(b) There exist $d^{\prime}, \delta>0$ and a positive integer $k^{\prime}$ such that, for all finite fields $K$ of characteristic $\neq 2$, and for any $A \subset G(K)$ a set of generators of $G(K)$ satisfying $|A \cdot A \cdot A| \leq d|A|^{1+\epsilon}$ for some $d, \epsilon>0$, we have $\left|\operatorname{cl}\left(A^{\prime}\right)\right| \leq\left(d^{\prime} d^{\delta}\right)\left|A_{k^{\prime}}\right|^{\frac{1}{n+1}+\delta \epsilon}$.

Note that the Tripling Lemma (Lem.1.1) implies that similar bounds apply to any set $A_{l}$ where $l \ll_{n} 1$; for instance, if $|A \cdot A \cdot A| \ll\left|A_{l}\right|^{1+\epsilon}$ and $l \ll_{n} 1$, then $\left|A_{l} \cdot A_{l} \cdot A_{l}\right| \ll\left|A_{l}\right|^{1+O_{l}(\epsilon)}$, and so

$$
\left|\operatorname{cl}\left(A_{l}^{\prime}\right)\right| \ll_{l, n}\left|A_{k l}\right|^{\frac{1}{n+1}+O_{l, n}(\epsilon)} \ll_{l, n}|A|^{\frac{1}{n+1}+O_{l, n}(\epsilon)} .
$$

We need to be sure that the intersection of $A_{k_{\dagger}}$ with a rich torus $T$ does not lie inside a subtorus (of a certain kind).

Proposition 2.3. [He2, Cor. 5.14] Let $G=S L_{n}$ and let $\left(a_{1}, \ldots, a_{r}\right)$ be a tuple of nonnegative integers. Then there exists $C>0$ and a positive integer $k$ such that for a finite field $K$ with characteristic $>n$; for $T$ a maximal torus of $G$ defined over $\bar{K}$; for $\alpha: T \rightarrow \mathbb{A}^{1}$ a character of $T$ with kernel $T^{\prime}$ satisfying $\operatorname{deg}\left(T^{\prime}\right)=\left(a_{1}, \ldots, a_{r}\right)$; and for $A \subset G(K)$ a set of generators of $G(K)$, we have

$$
\left|A \cap T^{\prime}(K)\right| \leq C\left|A_{k}\right|^{\frac{1}{n+2}} .
$$

The significance of this special type of subtorus is explained by the next result.

Lemma 2.4. [He2, Lem.'s 4.2 and 4.14] Let $K$ be a field. Let $T / \bar{K} \subset\left(\mathrm{GL}_{1}\right)^{n}$ be a torus. Let $H$ be a subgroup of $T(\bar{K})$ that is contained in an algebraic subvariety $V$ of $T$ of positive codimension.

Then $H$ is contained in the kernel of a non-trivial character $\alpha: T \rightarrow G L_{1}$ whose exponents are bounded in terms of $n$ and $\overrightarrow{d e g}(V)$ alone.

2.1. Further results on conjugacy. We need several more ideas concerning conjugacy classes in $G$. An element $g$ of a linear algebraic group $G$ is said to be regular if $\operatorname{dim}\left(C_{G}(g)\right)$ is equal to the rank of $G$. When $G$ is connected, and $g$ is semisimple this is equivalent to requiring that $C_{G}(g)$ is a torus. When $G=S L_{n}$, an element is regular semisimple if and only if its eigenvalues are distinct.

Lemma 2.5. [He2, Lem. 5.9] Let $G=S L_{n}$ and $K$ be a field. Then there is a subvariety $W / K$ of $G$ of positive codimension and degree $\overrightarrow{\operatorname{deg}}(W)=\left(a_{1}, \ldots, a_{d}\right)$ such that $a_{1}, \ldots a_{d}, d<n^{2 n}$, and every element $g \in G(K)$ not on $W$ is regular semisimple.

Of course, since $W$ is a subvariety of $S L_{n}$ of positive codimension, we may apply the results of $₫ 1.2$, and escape from it. Escaping from a subvariety $W$ of a torus is only slightly harder, provided that the torus is rich.

Lemma 2.6. Let $G=S L_{n}$ and let $\left(w_{1}, \ldots, w_{d}\right)$ be a tuple of non-negative integers. There exists a positive integer $l$ and $\epsilon_{0}, c>0$ such that

(a) for all finite fields $K$ of characteristic $>n$; 
(b) for any $A$ a set of generators for $G$ satisfying $|A \cdot A \cdot A| \leq d|A|^{1+\epsilon}$ for some $d, \epsilon>0$ with $\epsilon<\epsilon_{0}$;

(c) for any corresponding rich torus $T\left(\right.$ so $\left.\left|A_{k} \cap T(K)\right| \geq\left(d^{\prime} d^{\delta}\right)|A|^{\frac{1}{n+1}-\delta \epsilon}\right)$;

(d) for any $W$ a proper subvariety of $T$ of degree $\left(w_{1}, \ldots, w_{d}\right)$;

we have

$$
\left|A_{l} \cap(T(K) \backslash W(K))\right| \geq c|A|^{\frac{1}{n+1}-\delta \epsilon} .
$$

Note that the value of $\delta$ given in the conclusion to Lem. 2.6 is fixed by Prop. 2.1 (as per the inequality written above at (ㄷ) ).

Proof. Suppose first that $\left\langle A_{l} \cap T(K)\right\rangle$ is not contained in $W(K)$. Then apply Prop. 1.2 with $A_{l} \cap T(K)$ instead of $A$, and $W$ instead of $V$, and the result follows.

Now suppose that $\left\langle A_{l} \cap T(K)\right\rangle \subseteq W(K)$. Then, by Lem. 2.4. $\left\langle A_{l} \cap T(K)\right\rangle$ is contained in the kernel of a non-trivial character $\alpha: T \rightarrow \mathbb{A}^{1}$ whose exponents are bounded in terms of $n$ and $\overrightarrow{\operatorname{deg}}(W)$ alone. Hence, by Prop. 2.3 with $A_{l}$ instead of $A$, and $l^{\prime}$ instead of $k$,

$$
\left|A_{l} \cap T(K)\right| \ll_{n, \overrightarrow{\operatorname{deg}}(W)}\left|A_{l l^{\prime}}\right|^{\frac{1}{n+2}} \ll|A|^{\frac{1}{n+2}+O_{n}(\epsilon)} .
$$

This contradicts the assumption that $T$ is a rich torus.

In what follows, we fix $\epsilon>0$, and fix $k_{\dagger}=\max \left\{k, k^{\prime}, l\right\}$ where $k$ and $k^{\prime}$ are as given in Prop. 2.2, and $l$ is as given in Lemma 2.6, for the latter result we take $W$ to be the variety defined in Lemma 2.5. Thus, for $A \subset G(K)$ a generating set that doesn't grow,

and for $T$ a rich torus, we have tight bounds on the intersection of $A_{k_{\dagger}}$ with the set of regular elements in $T(K)$, as well as on the number of regular conjugacy classes in $A_{k_{\dagger}}$.

Our analysis of conjugacy classes in $G$ will require one more definition: Given a matrix $g$ in $\mathrm{SL}_{n}(K)$, we define $\kappa(g) \in \mathbb{A}^{n-1}(K)$ to be the tuple

$$
\left(a_{n-1}, a_{n-2}, \ldots, a_{1}\right)
$$

of coefficients of

$$
\lambda^{n}+a_{n-1} \lambda^{n-1}+a_{n-2} \lambda^{n-2}+\ldots+a_{1} \lambda+(-1)^{n}=\operatorname{det}(\lambda I-g) \in K[\lambda]
$$

(the characteristic polynomial of $g$ ).

As is well-known, $\kappa(g)=\kappa\left(h g h^{-1}\right)$ for any $h$, i.e., $\kappa(g)$ is invariant under conjugation. If $g$ is a regular semisimple element of $\mathrm{SL}_{n}$ then $\kappa(g)$ actually determines the conjugacy class $\operatorname{cl}(g)$ of $g$.

\section{A NEW BOUnd ON SETS THAT DO NOT GROW}

Just as in Helfgott's work on $S L_{3}$, the trace is going to be important for us, although we will use it in a very different way.

3.1. Independence of traces. In this section we show that a certain set of linear forms is linearly dependent, and that any subset is linearly independent.

Let $m \in K\left[t_{1}, \ldots, t_{n}\right]$ be a monomial; we call $m$ square-free if $t_{j}^{2}$ does not divide $m$ for any $j=1, \ldots, n$. 
Lemma 3.1. 1 Let $s_{j}, j=1, \ldots, n$, be distinct elements of $K^{*}$. Then, for any $i, 0 \leq i \leq n$,

$$
A_{i}=\left(\begin{array}{ccccccc}
1 & s_{1} & \cdots & s_{1}^{i-1} & s_{1}^{i+1} & \cdots & s_{1}^{n} \\
1 & s_{2} & \cdots & s_{2}^{i-1} & s_{2}^{i+1} & \cdots & s_{2}^{n} \\
\vdots & \vdots & & \vdots & \vdots & & \vdots \\
1 & s_{n} & \cdots & s_{n}^{i-1} & s_{n}^{i+1} & \cdots & s_{n}^{n}
\end{array}\right)
$$

has determinant equal to

$$
\left(\prod_{1 \leq j<k \leq n}\left(s_{k}-s_{j}\right)\right) \times S_{n-i}
$$

where $S_{n-i}$ is the sum of all square-free monomials of degree $n-i$ in the variables $s_{1}, \ldots, s_{n}$. Proof. We prove the result by calculating a Vandermonde determinant in $\left\{s_{1}, \ldots, s_{n}, t\right\}$ for some variable $t$. We define

$$
f=\operatorname{det}\left(\begin{array}{cccc}
1 & s_{1} & \cdots & s_{1}^{n} \\
1 & s_{2} & \cdots & s_{2}^{n} \\
\vdots & \vdots & & \vdots \\
1 & s_{n} & \cdots & s_{n}^{n} \\
1 & t & \cdots & t^{n}
\end{array}\right)
$$

and consider $f$ as a polynomial of degree $n$ in $t$. Observe that $\operatorname{det} A_{i}=(-1)^{n+i} c_{i}$ where $c_{i}$ is the coefficient of $t^{i}$ in $f$.

The classical formula for a Vandermonde determinant implies that

$$
f=\prod_{1 \leq j<k \leq n}\left(s_{k}-s_{j}\right) \prod_{1 \leq k \leq n}\left(t-s_{k}\right) .
$$

Then $f=\left(\prod_{1 \leq j<k \leq n}\left(s_{k}-s_{j}\right)\right) g$ where $g=\prod_{1 \leq k \leq n}\left(t-s_{k}\right)$ is a polynomial of degree $n$ in $t$. Write $d_{i}$ for the coefficient of $t^{i}$ in $g$; to prove the lemma it is sufficient to observe that $d_{i}=(-1)^{n-i} S_{n-i}$.

Lemma 3.2. 2 Suppose that $t$ is a regular element of $T(K)$. For $i=0, \ldots, n$, define linear forms,

$$
l_{i}: G(K) \rightarrow K: g \mapsto \operatorname{tr}\left(t^{i} g\right) .
$$

These forms are linearly dependent. Furthermore, there exists a subvariety $W$ in $T$ of positive codimension and degree $\ll_{n} 1$, such that if $t \in T(K) \backslash W(K)$, then any subset of $n$ of these linear forms is linearly independent.

Proof. Write $T(K)$ as the group of $n \times n$ diagonal matrices over $K$ of determinant 1 . The linear forms $l_{i}$ are forms on $n$ variables, $g_{11}, \ldots, g_{n n}$. Since there are $n+1$ of these, they must be linearly dependent.

\footnotetext{
${ }^{1}$ We thank an anonymous referee for significantly simplifying the proof of this result.

${ }^{2} \mathrm{An}$ anonymous referee pointed out that this result follows directly from the Cayley-Hamilton identity; c.f. the proof of Lemma 5.1 .
} 
Now consider a set, $L$, of $n$ of these linear forms. Since $t$ is regular, all eigenvalues are distinct. Thus $L$ will be linearly independent provided a matrix of the form given in Lemma 3.1 is non-zero.

For $i, j=1, \ldots, n$ with $i<j$ define $V_{i, j}$ to be the variety defined by $g_{i i}-g_{j j}=0$, and let $V_{-1}=\bigcup_{1 \leq i<j \leq n} V_{i, j}$. Thus $V(K)$ contains all elements of $T(K)$ that are not regular.

Next define $W_{i}$ to be the variety defined by the equation $S_{i}=0$, where $S_{i}$ is as in Lemma 3.1] let $W_{-1}=\cup_{i=0}^{n} W_{i}$ and let $W=V_{-1} \cup W_{-1}$.

Clearly $V_{i, j}, i, j=1, \ldots, n$ with $i<j$, and $W_{i}, i=0, \ldots, n$ are subvarieties of $T$ of positive codimension. Then Lemma 3.1 yields the result.

3.2. A new bound. Now we work towards proving a new bound on generating sets that "do not grow." We begin with some notation. Fix $t$ an element of a torus $T(K)$ in $G(K)$, $i$ some integer satisfying $0 \leq i \leq n$. For $g$ an element of $G(K)$, define two $n$-tuples as follows:

$$
\begin{aligned}
\operatorname{Tr}_{\widehat{i}, t}(g) & =\left(\operatorname{tr}(g), \operatorname{tr}(t g), \ldots, \widehat{\operatorname{tr}\left(t^{i} g\right)}, \ldots, \operatorname{tr}\left(t^{n} g\right)\right) ; \\
\mathrm{Cl}_{\hat{i}, t}(g) & =\left(\operatorname{cl}(g), \operatorname{cl}(t g), \ldots, \widehat{\operatorname{cl}\left(t^{i} g\right)}, \ldots, \operatorname{cl}\left(t^{n} g\right)\right)
\end{aligned}
$$

For a set $A \subset G(K)$, define

$$
\begin{aligned}
& \operatorname{Tr}_{\widehat{i}, t}(A)=\left\{\operatorname{Tr}_{\widehat{i}, t}(g) \mid g \in A\right\} \\
& \mathrm{Cl}_{\hat{i}, t}(A)=\left\{\mathrm{Cl}_{\widehat{i}, t}(g) \mid g \in A\right\} \\
& \mathrm{Cl}_{\hat{i}, t}^{\prime}(A)=\left\{\mathrm{Cl}_{\widehat{i}, t}(g) \mid g \in A, \text { and } g, t g, \ldots, t^{n} g \text { are semisimple }\right\} .
\end{aligned}
$$

The inequality that we will prove relates to $\mathrm{Cl}_{\hat{i}, t}(A)$. In fact $\mathrm{Cl}_{\hat{i}, t}(A)$ corresponds (generically) to $\mathrm{cl}_{T(K)}(A)$, the set of $T(K)$-conjugacy classes of $G(K)$ that intersect $A$. We will not need this correspondence in what follows.

Lemma 3.3. Let $G=S L_{n}$, and let $K$ be a field. For $h_{0}, t_{0}, \ldots, t_{n} \in G(\bar{K})$, define

$$
f_{h_{0}, t_{0}, \ldots, t_{n}}: G \rightarrow \mathbb{A}^{n^{2}-1}: g \mapsto\left(\kappa\left(h_{0} g\right), \kappa\left(t_{0} g\right), \ldots, \kappa\left(t_{n} g\right)\right) .
$$

Define

$$
f_{\widehat{i}, h_{0}, t_{0}, \ldots, t_{n}}: G \rightarrow \mathbb{A}^{n^{2}-n}: g \mapsto\left(\kappa\left(h_{0} g\right), \kappa\left(t_{0} g\right), \ldots, \widehat{\kappa\left(t_{i} g\right)}, \ldots \kappa\left(t_{n} g\right)\right)
$$

Let $T / \bar{K}$ be a maximal torus of $G$. Then there exists $h_{0} \in G(\bar{K}), t \in T(\bar{K})$, and $g_{0} \in$ $G(\bar{K})$, such that the derivative of $f_{\widehat{i}, h_{0}, t, t^{2} \ldots, t^{n}}$ at $g=g_{0}$ is a nonsingular linear map.

This result should be compared with [He2, Lemma 5.5]; the method of proof is very similar. 
Proof. We may write the elements of $G(\bar{K})$ so that the elements of $T(\bar{K}) \subset G(\bar{K})$ become diagonal matrices. Let

$$
g_{0}=\left(\begin{array}{cccccc}
0 & 1 & 0 & \cdots & 0 & 0 \\
0 & 0 & 1 & \cdots & 0 & 0 \\
\vdots & \vdots & \vdots & \vdots & \vdots & \vdots \\
0 & 0 & 0 & \cdots & 1 & 0 \\
0 & 0 & 0 & \cdots & 0 & 1 \\
(-1)^{n-1} & 0 & 0 & \cdots & 0 & 0
\end{array}\right)
$$

Let $\vec{r}=\left(r_{1}, r_{2}, \ldots, r_{n}\right)$ be a vector in $\bar{K}^{n}$ with $r_{1} \cdot r_{2} \cdots r_{n}=1$. Define

$$
h_{1}=\left(\begin{array}{cccc}
r_{1} & 0 & \ldots & 0 \\
0 & r_{2} & \ldots & 0 \\
\vdots & \vdots & \vdots & \vdots \\
0 & 0 & \ldots & r_{n}
\end{array}\right)
$$

Let us look, then, at the derivative at $g=I$ of $g \mapsto \kappa\left(h_{1}^{j} g_{0} g\right)$ for $0 \leq j \leq n$. The derivative at $g=I$ of the map taking $g$ to the coefficient of $\lambda^{n-1}$ in $\operatorname{det}\left(\lambda I-h_{1}^{j} g_{0} g\right)$ (i.e., to $(-1)$ times the trace of $\left.h_{1}^{j} g_{0} g\right)$ is equal to the map taking each matrix $\gamma$ in the tangent space $\mathfrak{g}$ to $G$ at the origin to

$$
(-1) \cdot\left(r_{1}^{j} \gamma_{2,1}+r_{2}^{j} \gamma_{3,2}+\ldots+r_{k}^{j} \gamma_{k+1, k}+\ldots+(-1)^{n-1} r_{n}^{j} \gamma_{1, n}\right),
$$

where we write $\gamma_{k, l}$ for the entries of the matrix $\gamma$.

The derivative at $g=I$ of the map taking $g$ to the coefficient of $\lambda^{n-2}$ in $\operatorname{det}\left(\lambda I-h_{1}^{j} g_{0} g\right)$ is the map taking each $\gamma$ in $\mathfrak{g}$ to

$$
\begin{aligned}
(-1) \cdot\left(r_{1}^{j} r_{2}^{j} \gamma_{3,1}\right. & +r_{2}^{j} r_{3}^{j} \gamma_{4,2}+\ldots+r_{k}^{j} r_{k+1}^{k} \gamma_{k+2, k}+\ldots+r_{n-3}^{j} r_{n-2}^{j} \gamma_{n-1, n-3} \\
& \left.+r_{n-2}^{j} r_{n-1}^{j} \gamma_{n, n-2}+r_{n-1}^{j} \cdot(-1)^{n-1} r_{n}^{j} \gamma_{1, n-1}+(-1)^{n-1} r_{n}^{j} r_{1}^{j} \gamma_{2, n}\right) .
\end{aligned}
$$

In general, for $1 \leq l \leq n-1$, the derivative at $g=I$ of the map taking $g$ to the coefficient of $\lambda^{n-l}$ in $\operatorname{det}\left(\lambda I-h_{1}^{j} g_{0} g\right)$ is the map taking $\gamma$ to

$$
(-1) \sum_{k=1}^{n-k}\left(r_{k}^{j} \cdot r_{\underline{k+1}}^{j} \cdots r_{\underline{k+l-1}}^{j}\right) \cdot \gamma_{\underline{k+l, k}}+(-1)^{n} \sum_{k=n-l+1}^{n}\left(r_{k}^{j} \cdot r_{\underline{k+1}}^{j} \cdots r_{\underline{k+l-1}}^{j}\right) \cdot \gamma_{\underline{k+l, k}}
$$

where by $\underline{a}$ we mean the only element of $\{1,2, \ldots, n\}$ congruent to $a$ modulo $n$.

We see that the entries of $\gamma$ present in (3.2) are disjoint for distinct $1 \leq l \leq n-1$ (and disjoint from $\left\{\gamma_{1,1}, \gamma_{2,2}, \ldots, \gamma_{n, n}\right\}$, which would appear for $l=0$ ). Now, for $l$ fixed, (3.2) gives us a linear form on $n$ variables $\gamma_{k+l, k}$ for each $\leq j \leq n$. Let us check that, for every $1 \leq l \leq n-1$, any $n$ of these linear forms are linearly independent, provided that $\vec{r}$ was chosen correctly.

This is the same as checking that the $n-1$ determinants

$$
\left|\left(r_{k}^{j} \cdot \underline{r_{k+1}^{j}} \cdots r_{\underline{k+l-1}}^{j}\right)\right|_{1 \leq k \leq n, 0 \leq j \leq n, j \neq i}
$$

for $1 \leq l \leq n-1$ are non-zero for some choice of $r_{1}, r_{2}, \ldots, r_{n}$ with $r_{1} \cdot r_{2} \cdots r_{n}=1$. (What we really want to check is that the determinant (3.3) is non-zero after all signs in some 
columns are flipped; since those flips do not affect the absolute value of the determinant, it is just as good to check that the determinant (3.3) itself is non-zero.)

These are generalised Vandermonde determinants of the type studied in Lemma 3.1. that result implies that they have absolute value equal to

$$
\prod_{k_{1}<k_{2}}\left(r_{k_{2}} \cdot \underline{r_{k_{2}+1}} \cdots \underline{r_{k_{2}+l-1}}-r_{k_{1}} \cdot r_{\underline{k_{1}+1}} \cdots r_{\underline{k_{1}+l-1}}\right) \times S_{n-i}
$$

where $S_{n-i}$ is the sum of all square-free monomials $m$ of degree $n-i$ in the variables $r_{k} \cdot r_{k+1} \cdots r_{k+l-1}$ for $k=1, \ldots, n$.

For any given $k_{1}, k_{2} \in\{1, \ldots, n\}$ with $k_{1} \neq k_{2}$, and $l \in\{1, \ldots, n-1\}$, there exist $r_{1}, r_{2}, \ldots, r_{n} \in \bar{K}$ with $r_{1} r_{2} \cdots r_{n}=1$ such that $r_{k_{1}} \cdot r_{k_{1}+1} \cdots r_{k_{1}+l-1} \neq r_{k_{2}} \cdot r_{k_{2}+1} \cdots r_{k_{2}+l-1}$.

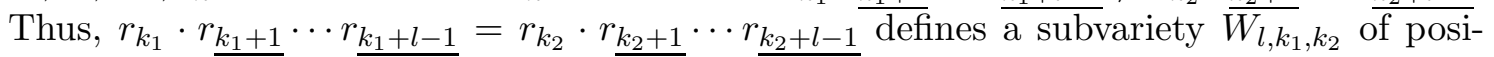
tive codimension in the (irreducible) variety $\overline{V \subset} \mathbb{A}^{n}$ of all tuples $\left(r_{1}, r_{2}, \cdots, r_{n}\right)$ with $r_{1} r_{2} \cdots r_{n}=1$.

In addition, for $i, j=1, \ldots, n$ with $i<j$, define $V_{i, j}$, the subvariety of $V$ given by the equation $r_{i}=r_{i}$; clearly the set $V \backslash V_{i, j}(\bar{K})$ is non-empty, and so these varieties are also of positive codimension in $V$. Then

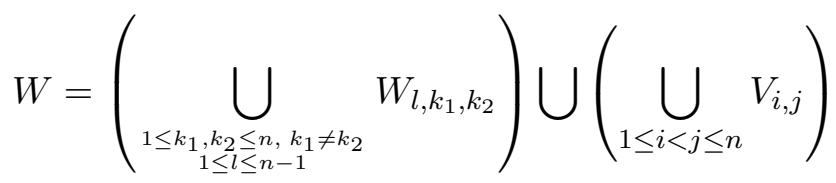

is a finite union of subvarieties of $V$ of positive codimension. Take $\vec{r}$ to be any point of $V(\bar{K})$ outside $W(\bar{K})$. It remains to choose $h_{0}$ so that the derivative of

$$
g \mapsto \kappa\left(h_{0} g\right)
$$

at $g=I$ is a linear map of full rank on the diagonal entries $\gamma_{1,1}, \gamma_{2,2} \ldots, \gamma_{n-1, n-1}$ of $\mathfrak{g}$. Let

$$
h_{0}=\left(\begin{array}{cccc}
t_{1} & 0 & \ldots & 0 \\
0 & t_{2} & \ldots & 0 \\
\vdots & \vdots & \vdots & \vdots \\
0 & 0 & \ldots & t_{n}
\end{array}\right),
$$

where $t_{1}, t_{2}, \ldots, t_{n} \in \bar{K}$ fulfil $t_{1} t_{2} \cdots t_{n}=1$. Then the derivative at $g=I$ of the map taking $g$ to the coefficient of $\lambda^{n-1}$ in $\operatorname{det}\left(\lambda I-h_{0} g\right.$ ) (i.e., to $(-1)$ times the trace of $h_{0} g$ ) equals the map taking $\gamma$ to

$$
(-1) \cdot\left(t_{1} \gamma_{1,1}+t_{2} \gamma_{2,2}+\cdots+t_{n} \gamma_{n, n}\right) .
$$

In general, the derivative of the map taking $g$ to the coefficient of $\lambda^{n-k}(1 \leq k \leq n-1)$ in $\operatorname{det}\left(\lambda I-h_{0} g\right)$ equals the map taking $\gamma$ to

$$
(-1)^{k} \cdot\left(c_{k, 1} \gamma_{1,1}+c_{k, 2} \gamma_{2,2}+\cdots+c_{k, n} \gamma_{n, n}\right)
$$

where $c_{k, i}$ is the sum of all monomials $t_{j_{1}} t_{j_{2}} \ldots t_{j_{k}}, 1 \leq j_{1}<j_{2}<\cdots<j_{k} \leq n$, such that one of the indices $j_{l}$ equals $i$. (For example, $c_{2,1}=t_{1} \cdot\left(t_{2}+t_{3}+\cdots+t_{n}\right)$.) Thus, our task is to find for which $t_{1}, t_{2}, \ldots, t_{n}$ the determinant

$$
\left|c_{i, j}-c_{i, n}\right|_{1 \leq i, j \leq n-1}
$$


is non-zero. Clearly, this will happen precisely when

$$
\left|c_{i-1, j}\right|_{1 \leq i, j \leq n} \neq 0,
$$

where we adopt the (sensible) convention that $c_{0, j}=1$ for all $j$.

A brief computation gives us that

$$
\left|c_{i-1, j}\right|_{1 \leq i, j \leq n}=(-1)^{\lfloor n / 2\rfloor} \cdot\left|t_{j}^{i-1}\right|_{1 \leq i, j \leq n} .
$$

This is a Vandermonde determinant; it equals $\prod_{j_{1}<j_{2}}\left(t_{j_{2}}-t_{j_{1}}\right)$. The equation $t_{j_{2}}=t_{j_{1}}$ defines a subvariety of positive codimension in the variety $V \subset \mathbb{A}^{n}$ of all $t_{1}, t_{2}, \ldots, t_{n}$ with $t_{1} t_{2} \cdots t_{n}=1$. Thus, we may choose $t_{1}, t_{2}, \ldots, t_{n}$ such that $t_{1} t_{2} \cdots t_{n}=1$ and $\prod_{j_{1}<j_{2}}\left(t_{j_{2}}-t_{j_{1}}\right) \neq 0$.

We are now able to prove the proposition that we have been aiming for (c.f. [He2, Prop. 5.15]).

Proposition 3.4. Let $G=\mathrm{SL}_{n}$. There exists a positive integer $k$ and $c, e>0$ such that for any $\left(a_{1}, \ldots, a_{d}\right)$, a tuple of non-negative integers, there exists a positive integer $k^{\prime}$ and $c^{\prime}>0$ such that

- for any $K$ a finite field such that $|K|<e$;

- for any $W / \bar{K}$, a proper subvariety of $G$, such that $\overrightarrow{d e g}(W)=\left(a_{1}, \ldots, a_{d}\right)$;

- for any $E \subset T(K)$ where $T / \bar{K}$ is a maximal torus of $G$.

- for any $A \subset G(K)$, a set of generators of $G(K)$; ;

one of the following holds

(a) there exists $h_{0} \in A_{k}$, and a subset $E^{\prime} \subset E_{k}$ with $\left|E^{\prime}\right| \geq c|E|$ such that, for each $t \in E^{\prime}$, there are $\geq c^{\prime}|A|$ distinct tuples

$$
\left(\kappa\left(h_{0} g\right), \kappa(g), \kappa(t g), \kappa\left(t^{2} g\right), \ldots, \widehat{\kappa\left(t^{i} g\right)}, \ldots, \kappa\left(t^{n} g\right)\right) \in \mathbb{A}^{n^{2}-1}(K)
$$

with $g \in A_{k^{\prime}}$ satisfying $h_{0} g, g, t g, t^{2} g, \ldots, t^{n} g \notin W(K)$, or

(b) $E$ is contained in the kernel of a non-trivial character $\alpha: T \rightarrow G L_{1}$ whose exponents are bounded in terms of $n$ alone.

Proof. Let $X=G \times T$ and $Y=\left(\mathbb{A}^{n-1}\right)^{n+1}=\mathbb{A}^{n^{2}-1}$. Let $f: X \times G \rightarrow Y$ be given by

$$
f\left(\left(h_{0}, t\right), g\right)=\left(\kappa(h g), \kappa(g), \kappa(t g), \kappa\left(t^{2} g\right), \ldots, \widehat{\kappa\left(t^{i} g\right)}, \ldots, \kappa\left(t^{n} g\right)\right) .
$$

Let $Z_{X \times G}$ be as in Lemma 1.6, then $\overrightarrow{\operatorname{deg}}\left(Z_{X \times G}\right) \ll_{n} 1$. Thanks to Lem. 3.3, we know $Z_{X \times G}$ is a proper subvariety of $X \times G$.

Let $Z_{G \times T \times G}$ be $Z_{X \times G}$ under the identification $G \times T \times G=X \times G$; write the elements of $Z_{G \times T \times G}$ in the form $\left(h_{0}, t, g\right)$. By the argument in [He2, $\left.\S 2.5 .3\right]$, there is a proper subvariety $Z_{G} \subset G$ (with $\overrightarrow{\operatorname{deg}}\left(Z_{G}\right) \ll \overrightarrow{\operatorname{deg}}\left(Z_{G \times T \times G}\right)$, and so $\overrightarrow{\operatorname{deg}}\left(Z_{G}\right) \ll_{n} 1$ ) such that, for all $h_{0} \in G(\bar{K})$ not on $Z_{G}$, the fibre $\left(Z_{G \times T \times G}\right)_{h=h_{0}}$ is a proper subvariety of $T \times G$.

By escape (Lem. 1.4, it is here that that $|K| \gg_{n} 1$ is used), there is an $h_{0} \in A_{k}, k \ll_{n} 1$, such that $h_{0}$ lies outside $Z_{G}$; thus, by the definition of $Z_{G}$, the fibre $V_{T \times G}:=\left(Z_{G \times T \times G}\right)_{h=h_{0}}$ is a proper subvariety of $T \times G$. Again by [He2, $\S 2.5 .3]$, there is a proper subvariety $V_{T}$ with $\overrightarrow{\operatorname{deg}}\left(V_{T}\right) \ll \overrightarrow{\operatorname{deg}}\left(V_{T \times G}\right) 1$ (and so $\overrightarrow{\operatorname{deg}}\left(V_{T}\right) \ll_{n} 1$ ) such that, for all $t_{0} \in T(\bar{K})$ not on $V_{T}$, the fibre $\left(V_{T \times G}\right)_{t=t_{0}}$ is a proper subvariety of $G$. 
Suppose first that $\langle E\rangle \not \subset V_{T}(K)$. We may then use escape from subvarieties (Prop. 1.2 with $A=E, V=V_{T}(K)$ and $\left.G=\mathscr{O}=\langle E\rangle\right)$ to obtain a subset $E^{\prime} \subset E_{k}\left(k \ll_{n} 1\right)$ with $\left|E^{\prime}\right| \gg_{n}|E|$ and $E^{\prime} \subset T(K) \backslash V_{T}(K)$. Now consider any $t_{0} \in E^{\prime}$. The fibre $\left(V_{T \times G}\right)_{t=t_{0}}$ is a proper subvariety of $G$, and, since $W$ is a proper subvariety of $G$, we conclude that

$$
V^{\prime}=\left(V_{T \times G}\right)_{t=t_{0}} \cup h_{0}^{-1} W \cup W \cup t_{0}^{-1} W \cup \ldots \cup t_{0}^{-n} W .
$$

is a proper subvariety of $G$ as well (with $\overrightarrow{\operatorname{deg}}\left(V^{\prime}\right) \ll_{n, \overrightarrow{\operatorname{deg}}(W)} 1$ ). We now recall the definition of $Z_{X \times G}$ (a variety outside which the map $f$ is non-singular) and use the result on non-singularity (Cor. 1.5 applied to the function $f_{h_{0}, t_{0}}: G \rightarrow Y$ given by $\left.f_{h_{0}, t_{0}}(g)=f\left(\left(h_{0}, t_{0}\right), g\right)\right)$ to obtain that

$$
\left|f_{h_{0}, t_{0}}\left(A_{k^{\prime}} \cap\left(G(K) \backslash V^{\prime}(K)\right)\right)\right| \gg_{n, \overrightarrow{\operatorname{deg}}(W)}|A|
$$

with $k^{\prime} \ll_{n, \overrightarrow{\operatorname{deg}}(W)} 1$. This gives us conclusion (国).

Suppose now that $\langle E\rangle \subset V_{T}(K)$. By Lemma 2.4, we obtain that $E$ is contained in the kernel of a non-trivial character $\alpha: T \rightarrow \mathbb{A}^{1}$ whose exponents are $\ll_{n} 1$.

Given our generating set $A$ satisfying $|A \cdot A \cdot A| \ll_{n}|A|^{1+\epsilon}$, recall that Prop. 2.1 implies that there exists a torus $T$ that is rich. In other words

$$
\left|A_{k} \cap T(K)\right| \gg|A|^{\frac{1}{n+1}}-O_{n}(\epsilon)
$$

for some $k \ll_{n} 1$. In fact, by Lem. 2.6 we know that when $T$ is rich, the set of regular elements in $A_{k_{\dagger}} \cap T(K)$ has size $\gg|A|^{\frac{1}{n+1}}-O_{n}(\epsilon)$, where $k_{\dagger} \ll_{n} 1$. These facts allow us to prove the following corollary.

Corollary 3.5. Let $\epsilon>0$ and suppose that $\epsilon$ is smaller than some constant $\epsilon_{0}$ depending only on $n$. Let $A$ be a generating set such that $|A \cdot A \cdot A| \ll_{n}|A|^{1+\epsilon}$, and let $T$ be a rich maximal torus. Then there are integers $k, l \ll_{n} k_{\dagger}$ and a set $E^{\prime}$ in $A_{k} \cap T(K)$ such that $\left|E^{\prime}\right| \gg|A|^{\frac{1}{n+1}+O_{n}(\epsilon)}$ and

$$
|A|^{\frac{n}{n+1}-O_{n}(\epsilon)} \ll_{n}\left|\mathrm{Cl}_{i, t}^{\prime}\left(A_{l}\right)\right|,
$$

for all $t \in E^{\prime}$. Moreover there are $\gg|A|$ elements of $A_{l}$ such that $g, t g, \ldots, t^{n} g$ are semisimple.

Proof. Let $W$ be the variety of elements in $G$ that are not semisimple, and note that, by Lem. 2.5, $\overrightarrow{\operatorname{deg}}(W) \ll_{n} 1$. Set $E=A_{k_{\dagger \dagger}} \cap T(K)$ where $T$ is a rich torus and $k_{\dagger \dagger}=\max \left\{k_{\dagger}, k\right\}$ for $k$ the constant given in Prop. 3.4, thus Prop. 2.1 implies that $|E| \gg_{n}|A|^{\frac{1}{n+1}-O_{n}(\epsilon)}$. Now apply Prop. 3.4 to $A$. Since we are assuming that $|A \cdot A \cdot A| \ll_{n}|A|^{1+\epsilon}$, we may assume that $K$ is larger than some constant depending only on $n$ (otherwise our assumption is trivially violated). Thus we lie in situation (a) or (b) of Prop. 3.4.

Suppose first that we are in situation (b). Then Lem. 2.4 and Prop. 2.3 imply that $|E| \ll_{n}\left|A_{l}\right|^{\frac{1}{n+2}}$ where $l \ll_{n} 1$. For $\epsilon$ small enough with respect to $n$ this gives a contradiction.

Thus we are in situation (a) and observe first that

$$
\left|E^{\prime}\right| \gg_{n}|E| \gg_{n}|A|^{\frac{1}{n+1}-O_{n}(\epsilon)}
$$


as required. Now Prop. 2.2 implies that that there are at most $\mid A_{k_{\dagger \dagger}}{ }^{\frac{1}{n+1}+O_{n}(\epsilon)} \ll_{n}$ $|A|^{\frac{1}{n+1}+O_{n}(\epsilon)}$ distinct tuples $\kappa(g)$, for $g$ a semisimple element in $A$. Indeed the same upper bound holds for tuples $\kappa\left(h_{0} g\right)$, for $g \in A$ such that $h_{0} g$ is simple. Prop. 3.4 implies, therefore, that there are $\gg_{n}|A|^{1-\frac{1}{n+1}-O(\epsilon)}$ elements in $\mathrm{Cl}_{i, t}^{\prime}(A)$ as required.

The final statement of the corollary follows immediately from Prop. 3.4.

\section{Moving From CONJUGaCy Classes to tRACES}

Cor. 3.5 gives us a bound on the size of the set $\mathrm{Cl}_{i, t}^{\prime}\left(A_{l}\right)$. In order to prove that small sets grow we will need to work with the tuple $\operatorname{Tr}_{i, t}^{\prime}\left(A_{l}\right)$. To make the transfer from conjugacy classes to traces we introduce the concept of wealth. Fix $l_{\dagger}$, the value for $l$ given by Cor. 3.5, $t$ a regular semisimple element; and $i$ an element of $\{0, \ldots, n\}$. The wealth of an element $r$ of $K$ is defined to be

$$
\diamond_{t, i}(r)=\mid\left\{\operatorname{cl}(g): \operatorname{tr}\left(t^{i} g\right)=r, g \in A_{l_{\dagger}} \text { and } t^{i} g \text { is semisimple }\right\} \mid .
$$

Now fix $\epsilon$ a small positive number; for $t \in A_{k_{\dagger}} \cap T(K)$ we define $A_{t,\left(j_{0}, j_{1}, \ldots, j_{n}\right)}$ where $\left(j_{0}, \ldots, j_{n}\right)$ is a tuple of integers:

$$
\begin{aligned}
A_{t,\left(j_{0}, j_{1}, \ldots, j_{n}\right)}=\left\{g \in A_{l_{+}} \mid\right. & 2^{j_{0}}<\diamond_{t, 0}(\operatorname{tr}(g)) \leq 2^{j_{0}+1} \\
& 2^{j_{1}}<\diamond_{t, 1}(\operatorname{tr}(t g)) \leq 2^{j_{1}+1}, \ldots, \\
& 2^{j_{n}}<\diamond_{t, n}\left(\operatorname{tr}\left(t^{n} g\right)\right) \leq 2^{j_{n}+1}, \text { and } \\
& \left.g, t g, \ldots, t^{n} g \text { are semisimple }\right\} .
\end{aligned}
$$

Thus $A_{t,\left(j_{0}, j_{1}, \ldots, j_{n}\right)}$ is a set of elements in $A_{l_{\dagger}}$ such that the corresponding tuple of traces of each element represents a roughly constant number of conjugacy classes. We often write $A_{t, j}$ for $A_{t,\left(j_{0}, \ldots, j_{n}\right)}$.

Note that this definition is dependent on our generating set $A$, on the constant $\epsilon$, and on the element $t$; note, furthermore, that there are at most $\left(\log _{2}|A|\right)^{n+1}$ tuples $\left(j_{0}, \ldots, j_{n}\right)$ such that $A_{t,\left(j_{0}, j_{1}, \ldots, j_{n}\right)}$ is non-empty.

Lemma 4.1. Suppose that $T$ is a rich torus and take $t \in T(K)$. Suppose that $\left|A_{t, \vec{j}}\right| \geq$ $|A|^{1-O(\epsilon)}$. Then

$$
2^{\max _{i} j_{i}-\min _{i} j_{i}} \ll d|A|^{O(\epsilon)}
$$

provided that $t$ lies outside a proper subvariety $W \subset T$ of degree $\ll_{n} 1$.

Note that the subvariety $W$ will be defined so as to include all of the non-regular elements.

Proof. By Cor. 3.5, the number of different tuples $\operatorname{Tr}_{\widehat{i}, t}(g)$, as $g$ varies over $A_{t, \vec{j}}$, is at least

$$
\frac{\mathrm{Cl}_{i, t}^{\prime}(A)}{2^{j_{0}+\cdots+j_{i-1}+j_{i+1}+\cdots+j_{n}+n}} \gg_{n} \frac{|A|^{\frac{n}{n+1}}-O(\epsilon)}{2^{j_{0}+\cdots+j_{i-1}+j_{i+1}+\cdots+j_{n}+n}} .
$$

On the other hand

$$
\left|\left\{\operatorname{tr}\left(t^{i} g\right) \mid g \in A_{t, \vec{j}}, 2^{j_{i}}<\diamond_{t, i}\left(\operatorname{tr}\left(t^{i} g\right)\right) \leq 2^{j_{i}+1}\right\}\right| \leq \frac{\left|\operatorname{cl}\left(A_{i+l_{\dagger}}^{\prime}\right)\right|}{2^{j_{i}}} .
$$


So, by Prop. 2.2 the number of different tuples $\operatorname{Tr}_{\widehat{i}, t}(g)$ can take on is at most

$$
\frac{\left|\operatorname{cl}\left(A_{n+l_{\dagger}}^{\prime}\right)\right|^{n}}{2^{j_{0}+\cdots+j_{i-1}+j_{i+1}+\cdots+j_{n}}} \ll_{n} \frac{|A|^{\frac{n}{n+1}}+O_{n}(\epsilon)}{2^{j_{0}+\cdots+j_{i-1}+j_{i+1}+\cdots+j_{n}}} .
$$

Now we need a definition:

$$
\operatorname{Tr}_{\widehat{i}, \widehat{k}, t}(g)=\left(\operatorname{tr}(g), \operatorname{tr}(t g), \ldots, \widehat{\operatorname{tr}\left(t^{i} g\right)}, \ldots, \widehat{\operatorname{tr}\left(t^{k} g\right)}, \ldots, \operatorname{tr}\left(t^{n} g\right)\right) .
$$

Again the number of different tuples $\operatorname{Tr}_{\hat{i}, \widehat{k}, t}(g)$ as $g$ varies over $A_{t, \vec{j}}$ is at most

$$
\frac{\left|\operatorname{cl}\left(A_{n k+l_{\dagger}}^{\prime}\right)\right|^{n-1}}{2^{j_{0}+\cdots+j_{i-1}+j_{i+1}+\cdots+j_{k-1}+j_{k+1}+\cdots+j_{n}}} \ll_{n} \frac{|A|^{\frac{n-1}{n+1}+O_{n}(\epsilon)}}{2^{j_{0}+\cdots+j_{i-1}+j_{i+1}+\cdots+j_{k-1}+j_{k+1}+\cdots+j_{n}}} .
$$

Then, for a given tuple $\vec{r}=\left(r_{0}, \ldots, r_{i-1}, r_{i+1}, \ldots, r_{k-1}, r_{k+1}, \ldots, r_{n}\right) \in K^{n-1}$ such that $r_{i}=\operatorname{tr}\left(t^{i} g\right)$ for some $g \in A_{t, \vec{j}}$, and a given index $k$, there are at least

$$
\begin{aligned}
& \gg_{n}\left(\frac{|A|^{\frac{n}{n+1}}-O(\epsilon)}{2^{j_{0}+\cdots+j_{i-1}+j_{i+1}+\cdots+j_{n}}}\right) \times\left(\frac{|A|^{\frac{n-1}{n+1}+O(\epsilon)}}{2^{j_{0}+\cdots+j_{i-1}+j_{i+1}+\cdots+j_{k-1}+j_{k+1}+\cdots+j_{n}}}\right)^{-1} \\
& =\frac{|A|^{\frac{1}{n+1}}-O(\epsilon)}{2^{j_{k}}}
\end{aligned}
$$

values for $\operatorname{tr}\left(t^{k} g\right)$ such that $\operatorname{Tr}_{\hat{i}, t}(g)=\left(r_{0}, \ldots, r_{i-1}, r_{i+1}, \ldots, r_{k-1}, \operatorname{tr}\left(t^{k} g\right), r_{k+1}, \ldots, r_{n}\right)$ for some $g \in A_{l_{\dagger}}$. Now we wish to apply Lem. 3.2, to do this we must assume, as we do, that $t$ lies outside an exceptional subvariety $W$ of bounded degree. Then Lem. 3.2 implies that there exist $a_{1}, \ldots, a_{k-1}, a_{k+1}, \ldots, a_{n} \in K$ such that, for all $g \in G(K)$,

$$
\operatorname{tr}\left(t^{k} g\right)=a_{1} \operatorname{tr}(g)+\cdots a_{k-1} \operatorname{tr}\left(t^{k-1} g\right)+a_{k+1} \operatorname{tr}\left(t^{k+1} g\right)+\cdots a_{n} \operatorname{tr}\left(t^{n} g\right) .
$$

We conclude, therefore, that (4.1) is a lower bound on values for $\operatorname{tr}\left(t^{i} g\right)$ such that

$$
\operatorname{Tr}_{\widehat{k}, t}(g)=\left(r_{1}, \ldots, r_{i-1}, \operatorname{tr}\left(t^{i} g\right), r_{i+1}, \ldots, r_{k-1}, r_{k+1}, \ldots, r_{n+1}\right) .
$$

Observe that there are at least $2^{j_{i}}$ values of $\operatorname{cl}\left(t^{i} g\right)$ for a fixed value of $\operatorname{tr}\left(t^{i} g\right)$. Thus

$$
|\operatorname{cl}(A)| \geq 2^{j_{i}} \frac{|A|^{\frac{1}{n+1}}-O(\epsilon)}{2^{j_{k}}} .
$$

Since $|\operatorname{cl}(A)| \ll|A|^{\frac{1}{n+1}+O(\epsilon)}$ we conclude that $2^{j_{i}-j_{k}} \ll|A|^{O(\epsilon)}$ as required.

\section{SMall SETS GROW}

We continue to analyse our set $A$ that "doesn't grow". Let $T$ be a rich torus; by the pigeonhole principle, every $t \in T(K)$ has a "popular tuple", $\vec{j}$, such that $\left|A_{t, \vec{j}}\right| \geq$ $|A|^{1-O(\epsilon)}$. In this case Lem. 4.1 implies that $\max _{i} j_{i}-\min _{i} j_{i} \ll O(\epsilon) \log _{2}|A|$. Define an integer

$$
p \rightarrow \vec{j}=\mid\left\{t \in T(K) \mid t \notin W(K) \text { and }\left|A_{t, \vec{j}}\right| \geq|A|^{1-O(\epsilon)}\right\} \mid
$$

where $W$ is as in Lem. 4.1,

Lem. 3.2 implies that, for $t \in T(K) \backslash W(K)$, the linear forms

$$
l_{i}: G(K) \rightarrow K, g \mapsto \operatorname{tr}\left(t^{i} g\right)
$$


are linearly dependent; what is more any subset of $n$ of these is linearly independent. These facts allow us to define

$$
f: T(K) \backslash W(K) \rightarrow K^{n}, t \mapsto \vec{r}=\left(r_{0}, \ldots, r_{n-1}\right)
$$

where $\vec{r}$ satisfies the equation

$$
\operatorname{tr}\left(t^{n} g\right)=r_{0} \operatorname{tr}(g)+r_{1} \operatorname{tr}(t g)+\cdots+r_{n-1} \operatorname{tr}\left(t^{n-1} g\right)
$$

for all $g \in G$.

We need to know that $f$ is, in some sense, close to being one-to-one. The following lemma gives the result that we need 3

Lemma 5.1. Suppose that $S$ is a subset of $T(\bar{K}) \backslash W(\bar{K})$, where $W$ is as above. Then

$$
|f(S)| \geq \frac{1}{n !}|S| \text {. }
$$

Proof. Recall the definition of $\kappa(t)=\left(a_{1}, \ldots, a_{n-1}\right)$ given at the end of Section 2. We apply the Cayley-Hamilton theorem (which states that a square matrix $t$ satisfies the polynomial $\operatorname{det}(\lambda I-t)=0)$ to conclude that

$$
\begin{aligned}
& \operatorname{det}(\lambda I-t)=\lambda^{n}+a_{n-1} \lambda^{n-1}+\cdots+a_{1} \lambda+(-1)^{n} ; \\
\Rightarrow & t^{n}=-a_{n-1} t^{n-1}-a_{n-2} t^{n-2}-\cdots-a_{1} t-(-1)^{n} ; \\
\Rightarrow & t^{n} g=-a_{n-1} t^{n-1} g-a_{n-2} t^{n-2} g-\cdots-a_{1} t g-(-1)^{n} g \text { for all } g \in G(K) ; \\
\Rightarrow & \operatorname{tr}\left(t^{n} g\right)=-a_{n-1} \operatorname{tr}\left(t^{n-1} g\right)-\cdots-a_{1} \operatorname{tr}(t g)-(-1)^{n} \operatorname{tr}(g) \text { for all } g \in G(K) ; \\
\Rightarrow & r_{n-1}=-a_{n-1}, r_{n-2}=-a_{n-2}, \ldots, r_{1}=-a_{1}, r_{0}=(-1)^{n+1} .
\end{aligned}
$$

Now, all elements in $T(\bar{K}) \backslash W(\bar{K})$ are regular; write $T(\bar{K})$ as the set of diagonal matrices in $G(\bar{K})$. Then two elements $g, h \in T(\bar{K}) \backslash W(\bar{K})$ satisfy $\kappa(g)=\kappa(h)$ if and only if there is an element $\sigma$ in the symmetric group on $n$ letters such that $g_{i i}=h_{\sigma(i) \sigma(i)}$. The result follows.

Now set $\overrightarrow{j^{\mathrm{max}}}=\left(j_{0}^{\max }, \ldots, j_{n}^{\max }\right)$ be the tuple for which $p \rightarrow$ is maximal (so $\overrightarrow{j^{\mathrm{max}}}$ is the "most popular" popular tuple). Define

$$
D=\left\{t \in T(K) \cap A_{k_{\dagger}} \mid t \notin V(K) \cup W(K) \text { and }\left|A_{t, \overrightarrow{j^{\max }}}\right| \geq|A|^{1-O(\epsilon)}\right\} .
$$

Observe that $|D|=p_{j_{\text {max }}}$. Since there are at most $\left(\log _{2}|A|\right)^{n}$ choices for $\overrightarrow{j^{\mathrm{max}}}$, Lem. 2.6 implies that $|D| \gg_{n}|A|^{\frac{1}{n+1}}-O(\epsilon)$.

We also define $j_{\max }^{\max }\left(\right.$ resp. $\left.j_{\min }^{\max }\right)$ to be the maximum (resp. minimum) element in $\left\{j_{0}^{\max }, \ldots, j_{n}^{\max }\right\}$.

We need the following result from arithmetic combinatorics; it is a strengthening of [He2, Cor. 3.8].

Proposition 5.2. There exists $c>0$ such that, for any $n>2$ a positive integer and any $\delta>0$, there exist $e, \eta>0$ such that

- for every prime $p$, and field $K=\mathbb{Z} / p \mathbb{Z}$;

\footnotetext{
${ }^{3}$ We thank an anonymous referee for significantly simplifying the proof of this result.
} 
- for every set $X \subset K$ such that $|X| \leq p^{1-\delta}$;

- for every set $Y \subset K^{n}$;

- for each $\vec{y} \in Y$, and $X_{\vec{y}} \subseteq X^{n}$ such that

$$
\vec{y} \cdot X_{\vec{y}}=\left\{y_{1} x_{1}+\cdots+y_{n} x_{n}: \vec{x} \in X_{\vec{y}}\right\}
$$

is contained in $X$;

either $|Y|<|X|^{\text {cn }}$ or

$$
\left|X_{\vec{y}}\right| \leq e|X|^{n-\eta} \text { for some } \vec{y} \in Y .
$$

Proof. Suppose $|Y| \geq|X|^{c n \eta}$ (c>0 to be chosen later) and $\left|X_{\vec{y}}\right| \gg|X|^{n-\eta}$ for every $\vec{y} \in Y$. We will try to derive a contradiction.

There is a coordinate $1 \leq i \leq n$ such that the image $\pi_{i}(Y)$ of $Y$ under the projection $\pi_{i}$ to the $i$ th coordinate has $\geq|Y|^{1 / n}$ elements. We can assume without loss of generality that $n=1$. Let $Y^{\prime} \subset Y$ be a maximal set such that $\left.\pi_{1}\right|_{Y^{\prime}}$ is injective. Then $\left|Y^{\prime}\right| \gg|Y|^{1 / n}$.

For $x_{3}, x_{4}, \ldots, x_{n} \in X$, let

$$
R_{x_{3}, x_{4}, \ldots, x_{n}}=\left\{\left(\vec{y}, x_{1}, x_{2}\right) \in Y^{\prime} \times X \times X:\left(x_{1}, x_{2}, \ldots, x_{n}\right) \in X_{\vec{y}}\right\} .
$$

By assumption, $\sum_{x_{3}, \ldots, x_{n} \in X}\left|R_{x_{3}, \ldots, x_{n}}\right| \gg\left|Y^{\prime}\right||X|^{n-\eta}$, and so we can fix $x_{3}, x_{4}, \ldots, x_{n} \in X$ such that $S=R_{x_{3}, \ldots, x_{n}}$ has $\gg\left|Y^{\prime}\right||X|^{2-\eta}$ elements. For $\vec{y} \in Y^{\prime}$, let $c_{\vec{y}}=x_{3} y_{3}+\ldots+x_{n} y_{n}$.

We have $S \subset Y^{\prime} \times X \times X$; let $S_{\vec{y}}=\left\{\left(x_{1}, x_{2}\right) \in X \times X:\left(\vec{y}, x_{1}, x_{2}\right) \in S\right\}$. There is an $\epsilon>0$ such that the sets $S_{\vec{y}}$ with $\left|S_{\vec{y}}\right| \leq \epsilon|X|^{2-\eta}$ contribute at most $\frac{1}{2}|S|$ elements to $S$. Let $Y^{\prime \prime}$ be the set of all $\vec{y} \in Y^{\prime}$ with $\left|S_{\vec{y}}\right|>\epsilon|X|^{2-\eta}$. Then $\sum_{\vec{y} \in Y}\left|S_{\vec{y}}\right| \geq \frac{1}{2}|S| \gg\left|Y^{\prime}\right||X|^{2-\eta}$ and so $\left|Y^{\prime \prime}\right| \gg\left|Y^{\prime}\right||X|^{\eta} \gg|X|^{(c-1) \eta}$. By the definition of $S_{\vec{y}}$,

$$
\left\{x_{1} y_{1}+x_{2} y_{2}+c_{\vec{y}}:\left(x_{1}, x_{2}\right) \in S_{\vec{y}}\right\} \subset X .
$$

Thus, for all $\vec{y} \in Y^{\prime \prime}$, there are $>\epsilon|X|^{2-\eta}$ distinct pairs $\left(x, x^{\prime}\right)=\left(x_{1}, x_{1} y_{1}+x_{2} y_{2}+c_{\vec{y}}\right) \epsilon$ $X \times X$ such that $x^{\prime}-y_{1} x \in y_{2} X+c_{\vec{y}}$. Thus the energy $E_{+}\left(X, y_{1} X\right)=\sum_{d \in X-y_{1} X} \mid\{a \in$ $\left.X, b \in y_{1} X: a-b=d\right\}\left.\right|^{2}$ is greater than $\frac{1}{\left|y_{2} X+c_{\vec{y}}\right|}\left(\epsilon|X|^{2-\eta}\right)^{2}=\epsilon^{2}|X|^{3-2 \eta}$. Hence

$$
\sum_{y_{1} \in \pi\left(Y^{\prime \prime}\right)} E\left(X, y_{1} X\right)>\epsilon^{2} \pi_{1}\left(Y^{\prime \prime}\right)|X|^{3-2 \eta}
$$

Recall that $\left.\pi_{1}\right|_{Y^{\prime}}$ is injective and $Y^{\prime \prime} \subset Y^{\prime}$ has $\gg|X|^{(c-1) \eta}$ elements; thus $\left|\pi_{1}\left(Y^{\prime \prime}\right)\right| \gg$ $|X|^{(c-1) \eta}$.

Hence, for $c$ and $p$ large enough and $\eta$ smaller than a constant times $\delta$, (5.2) is contradiction with Theorem $\mathrm{C}$ of $\mathrm{BO}$. (If $p$ is smaller than a constant, the statement we are trying to prove is trivially true.)

We are now able to prove our main result.

Main Theorem. Let $G=\mathrm{SL}_{n}$ and fix $\delta$ a positive number. Then there are positive numbers $\epsilon$ and $C$ such that, for all fields $K=\mathbb{Z} / p \mathbb{Z}$ (p prime), and all sets $A \subset G(K)$ that generate $G(K)$, either $|A|<p^{n+1-\delta}, \delta>0$ or $|A \cdot A \cdot A| \geq C|A|^{1+\epsilon}$. 
Proof. Suppose that $|A|<p^{n+1-\delta}$ and that $A$ does not grow; we seek a contradiction to Prop. 5.2. Let $\eta$ be some (small) positive number.

First set $R=K=\mathbb{Z} / p \mathbb{Z}$. We define $X$ to be the following set:

$$
\left\{\operatorname{tr}\left(t^{i} a\right) \mid t \in D, a \in A_{t, \overrightarrow{j^{\mathrm{max}}}}, i=0, \ldots, n ; \text { and } t^{i} a \text { is semisimple }\right\} .
$$

Observe that $|X| \leq \frac{\left|\operatorname{cl}\left(\left(A_{n k_{\dagger}+l_{\dagger}}\right)^{\prime}\right)\right|}{2^{j_{\min }^{\max }}}$ where $\left(A_{n k_{\dagger}+l_{\dagger}}\right)^{\prime}$ is the set of semisimple elements in $A_{n k_{\dagger}+l_{\dagger}}$. Prop. 2.2 implies that $\left|\operatorname{cl}\left(A_{n k_{\dagger}+l_{\dagger}}\right)^{\prime}\right| \ll_{n}|A|^{\frac{1}{n+1}+O(\epsilon)}$, and so

$$
|X| \leq \frac{|A|^{\frac{1}{n+1}}+O(\epsilon)}{2^{j_{\min }^{\max }}} .
$$

Thus if we pick $\epsilon$ small enough we know that $|X|<p^{1-\delta^{\prime}}$ for some positive number $\delta^{\prime}$, and we have satisfied the assumption in Prop. 5.2,

Now define $Y=f(D)$ and for $\vec{y} \in Y$ we define $X_{\vec{y}}$ to be the set of elements $\vec{x}$ in $X^{n}$ such that $\vec{y} \cdot \vec{x}$ is equal to the trace of a semisimple element in $D A$, i.e. such that $\vec{y} \cdot \vec{x}$ lies in $X$.

Our first job is to show that $\left|X_{\vec{y}}\right| \gg_{n}|X|^{n-\eta}$ for all $\vec{y} \in Y$. Take $\vec{y}=f(t)$ for some $t \in D$. Then $\vec{y}=\left(r_{0}, \ldots, r_{n-1}\right)$ where $\vec{r}$ satisfies the equation

$$
\operatorname{tr}\left(t^{n} g\right)=r_{0} \operatorname{tr}(g)+r_{1} \operatorname{tr}(t g)+\cdots+r_{n-1} \operatorname{tr}\left(t^{n-1} g\right)
$$

for all $g \in G$.

Now Prop. 3.4 implies that, for all $i=0, \ldots, n$,

$$
\left|\mathrm{Cl}_{\hat{i}, t}\left(A_{t, \overrightarrow{j^{\max }}}\right)\right| \gg_{n}\left|A_{t, \overrightarrow{j^{\max }}}\right|^{\frac{n}{n+1}+O(\epsilon)} \gg_{n}|A|^{\frac{n}{n+1}+O(\epsilon)} .
$$

Thus we conclude that

$$
\left|\operatorname{Tr}_{\hat{i}, t}\left(A_{t, \overrightarrow{j^{\max }}}\right)\right| \gg \frac{|A|^{\frac{n}{n+1}+O(\epsilon)}}{2^{j_{0}^{\max }+\cdots+j_{n}^{\max }+n}} .
$$

Next observe that Lem. 4.1 implies that the wealth for each entry in the tuple differs only by a factor of $O(\epsilon)$, hence we are able to conclude that

$$
\left|\operatorname{Tr}_{\hat{i}, t}(A)\right| \gg \frac{|A|^{\frac{n}{n+1}-O(\epsilon)}}{\left(2^{j_{\min }^{\max }}\right)^{n}} \gg|X|^{n-O(\epsilon)} .
$$

If we pick $\epsilon$ to be small enough in terms of $\eta$ then this implies that $\left|\operatorname{Tr}_{\hat{i}, t}(A)\right| \gg|X|^{n-\eta}$, as required.

Finally we need to show that $|Y| \gg|X|^{O(n \eta)}$. Now Lem. 5.1 implies that

$$
|Y|=|f(D)| \gg_{n}|A|^{\frac{1}{n+1}+O_{n}(\epsilon)} .
$$

We conclude that, provided we choose $\epsilon$ small enough with respect to $n$ and $\eta$, then $|Y| \gg_{n}|X|^{\eta}$. Thus we have a contradiction with Prop. 5.2 as required. 


\section{Generalisations}

We give a brief explanation as to how ideas from the current paper may be applied more generally. It should be a relatively straightforward matter to strengthen the Main Theorem to the following statement: for every $\delta>0$ there is an $\epsilon>0$ such that if $|A|<p^{n+1-\delta}$ or if $p^{n+1+\delta}<|A|<p^{2 n+2-\delta}$, then

$$
|A \cdot A \cdot A| \gg_{n}|A|^{1+\epsilon}
$$

where $\epsilon$ and the implied constant depend only on $n$.

To prove this statement, our current proof would need to be extended to consider vectors of the form

$$
\left(\begin{array}{c}
\operatorname{tr}(g) \\
\operatorname{tr}\left(g^{-1}\right)
\end{array}\right)
$$

for $g \in A$; analysis of the set of all such vectors would lead to a contradiction of a result similar to Prop. 5.2, but over the ring $K \times K$, rather than over $K$. This would be similar to the approach adopted in [He2, $\S 6]$, but would also need a concept of wealth for it to work in the current setting.

As it is we have restricted our attention simply to the set of traces, $\{\operatorname{tr}(g) \mid g \in A\}$. We did this because things are more transparent in this setting; but more importantly we did this because there seems no obvious way of extending the ideas in this paper to cover sets of size greater than $p^{2 n+2-\delta}$.

The significance of $\operatorname{tr}(g)$ and $\operatorname{tr}\left(g^{-1}\right)$ is that these are entries in the tuple $\kappa(g)$ (the first and last entry respectively). Both of these entries correspond to the fundamental $n$-dimensional representations of $G$ over $\bar{K}$; when $n=3$ these are all of the fundamental representations of $G$ and hence an analysis of these two representations gives information about sets of size all the way up to $|G|^{1-\delta}$.

When $n \geq 4$, however, the other entries in $\kappa$ correspond to representations of dimension strictly greater than $n$. This fact prevents us from using them as we use $\operatorname{tr}(g)$ and $\operatorname{tr}\left(g^{-1}\right)$.

The methods in the present paper seem to extend easily to $\mathrm{Sp}_{2 n}, n \geq 2$; there they show that $|A \cdot A \cdot A| \geq|A|^{1+\epsilon}$ for every $A \subset \operatorname{Sp}_{2 n}(K)$ with $|A|<p^{2 n+1-\delta}, \delta>0$. However, there is a technical reason why the extension of these methods to $\mathrm{SO}_{n}$ would be more problematic: both $\mathrm{SL}_{n}$ and $\operatorname{Sp}_{2 n}$ obey $(\operatorname{dim}(\pi)+1) \cdot \operatorname{dim}(T) \leq \operatorname{dim}(G)$, where $\pi$ is the smallest-dimensional faithful representation of $G$ and $T$ is the maximal torus of $G$. In contrast, in the case of $\mathrm{SO}_{n},(\operatorname{dim}(\pi)+1) \cdot \operatorname{dim}(T)=(2 m+1) \cdot m>\frac{n(n-1)}{2}=\operatorname{dim}(G)$ if $n=2 m$, and $(\operatorname{dim}(\pi)+1) \cdot \operatorname{dim}(T)=(2 m+2) \cdot m>\frac{n(n-1)}{2}=\operatorname{dim}(G)$ if $n=2 m+1$.

$$
* * *
$$

The difficulties in extending the result to $\mathrm{SO}_{n}$ complicate the following approach to proving that every subset $A \subset \mathrm{SL}_{n}(K)$ grows (as usual, $K=\mathbb{F}_{p}$ here). Let $A \subset \mathrm{SL}_{n}\left(\mathbb{F}_{p}\right)$, $|A| \geq p^{n-1+\delta}, \delta>0$ and consider the natural action of $S L_{n}(K)$ on $V=K^{n}$. Let $H$ be a maximal parabolic subgroup of $\mathrm{SL}_{n}\left(\mathbb{F}_{p}\right)$ stabilizing a one-dimensional subspace $\langle\vec{v}\rangle$ of $V$, i.e.,

$$
H=\left\{g \in \mathrm{SL}_{n}\left(\mathbb{F}_{p}\right): g \vec{v}=\lambda \vec{v} \text { for some } \lambda \in K^{*}\right\} .
$$

Then $[G: H] \ll p^{n-1}$, and so (by [He2, Lemma 7.2]), $\left|A^{-1} A \cap H\right| \gg p^{\delta}$. Then one can try to show that $A^{-1} A \cap H$ grows in $H$; from this, it would follow that $A$ itself grows $([\mathrm{He} 2$, 
Lemma 7.3]). The problem here is that we could have $\left\langle A^{-1} A \cap H\right\rangle \sim \mathrm{SO}_{n-1}$, for example. This possibility could be avoided if we could find a rich torus $T$ in $H$, or, conversely, if we could choose $H$ so that it contains a rich torus $T$.

The reason why this would be enough is the following. Suppose $\left\langle A^{-1} A \cap H\right\rangle \sim \mathrm{SO}_{n-1}$, or even $\left\langle A^{-1} A \cap H\right\rangle \sim K^{*} \times \mathrm{SO}_{n-1}$. The maximal torus of $K^{*} \times \mathrm{SO}^{n-1}$ has smaller dimension than that of $\mathrm{SL}_{n}$. Hence, since $A^{-1} A \cap T(K)$ is contained in $A^{-1} A \cap H \subset$ $\left\langle A^{-1} A \cap H\right\rangle \sim K^{*} \times \mathrm{SO}_{n-1}$, we cannot possibly have $\left|A_{k} \cap T(K)\right| \gg|A|^{\operatorname{dim}(T) / \operatorname{dim}(G)}$ (see Prop. 2.3 in the present paper), i.e., $T$ cannot be rich.

In general, by the same argument, if $T$ is rich and contained in $H$, then $A^{-1} A \cap H$ cannot be contained in any algebraic subgroup of $H$ of bounded degree whose maximal tori are of smaller dimension than $T$. The only connected algebraic subgroups $H^{\prime}$ of $H$ whose maximal tori are of the same dimension as a maximal torus of $\mathrm{SL}_{n}$ have form

$$
U \rtimes\left(\left(\mathrm{SL}_{m_{1}} \times \ldots \times \mathrm{SL}_{m_{k}}\right) T\right),
$$

where $U$ is a unipotent group, $m_{1}+m_{2}+\ldots+m_{k}=n$, and $T$ is a maximal torus.

It follows that $A^{-1} A \cap H$ is contained in one such group $H^{\prime}$, but not in any proper algebraic subgroup of $H^{\prime}$ of bounded degree. By [He2, Prop. 4.2], we conclude that $A^{-1} A \cap H$ is not contained in any algebraic subvariety of $H^{\prime}$ of bounded degree. Thus, we can work as if $A^{-1} A \cap H$ generated $H^{\prime}$. By induction on $n$, the theorem $|A \cdot A \cdot A| \geq|A|^{1+\epsilon}$ would then be proven for all $A \subset \mathrm{SL}_{n}\left(\mathbb{F}_{p}\right)$ of arbitrary size $|A| \leq\left|\mathrm{SL}_{n}\left(\mathbb{F}_{p}\right)\right|^{1-\delta}, \delta>0$.

The only gap in the above argument is that one must still prove that there exists a maximal parabolic subgroup $H$ containing a rich torus $T$. If a rich torus $T$ fixes some vector $\vec{v} \in K^{n}$, then $T$ lies in a maximal parabolic subgroup $H_{\vec{v}}$ of the required type. The only problem remaining, then, is to find a rich torus $T$ (i.e., a torus $T$ with $\left|A_{k} \cap T(K)\right| \gg$ $\left.|A|^{\frac{\operatorname{dim} T}{\operatorname{dim} G}-O(\epsilon)}\right)$ such that $T$ has at least one eigenvector in $K^{n}$ (as opposed to just in $\bar{K}^{n}$ ).

One possible approach to this last question is to look for regular semisimple elements in $A^{-1} A \cap H$, where $H$ is a maximal parabolic subgroup. If there is one such element, there will be many, and hence there will be a rich maximal torus $T$ inside $H$. If instead there are many non-semisimple elements, one can conjugate them by coset representatives of $H$ to produce $\gg|A|$ non-semisimple elements of $A_{k}$; this, in turn, results in $|A \cdot A \cdot A| \geq|A|^{1+\epsilon}$ by an argument sticking varieties in different directions (as in [He2, §4.3]). The only problem is that $A^{-1} A \cap H$ may consist almost entirely of non-regular semisimple elements, as then too many of their conjugates could be identical; a different argument would be needed in this case.

6.1. Final note. Shortly after the completion of this paper, and well after the completion of the research leading to it, Pyber and Szabo [PS], and Breuillard, Green, and Tao [BGT] independently announced that they had proven results more general than those in the present paper. According to these announcements, their methods are based in part on those of the second author [He1, He2], on which the present work also rests.

Funding. This work was supported by EPSRC (Engineering and Physical Sciences Research Council) grant EP/E054919/1.

Acknowledgements. We would like to thank R. Hill, M. Kassabov and N. Nikolov for interesting conversations on subgroups and tori in $\mathrm{SL}_{n}$. In addition, K. Raghavan, A. 
Zalesski and S. Murray made useful comments. We are likewise grateful for the hospitality of the people of Orsay (Paris Sud XI).

\section{REFERENCES}

[Bo] Bourgain, J. "Multilinear exponential sums in prime fields under optimal entropy condition on the sources." Geom. funct. anal. 18 (2009): 1477-1502.

[BG] Bourgain, J., and A. Gamburd. "Uniform expansion bounds for Cayley graphs of $\mathrm{SL}_{2}\left(\mathbb{F}_{p}\right)$. " Ann. of Math. 167 no. 2 (2008): 625-642.

[BGS] Bourgain, J., Gamburd, A., and P. Sarnak. "Affine linear sieve, expanders, and sum-product." Invent. Math., to appear.

[BGT] Breuillard, E., Green, B., and T. Tao. "Linear approximate groups." Preprint: arxiv.org:1001.4570.

[Di] Dinai, O. "Expansion properties of finite simple groups." Preprint.

[He1] Helfgott, H. A. "Growth and generation in $\mathrm{SL}_{2}(\mathbb{Z} / p \mathbb{Z})$." Ann. of Math. (2) 167, no. 2 (2008): 601-623.

[He2] Helfgott, H. A. "Growth in $\mathrm{SL}_{3}(\mathbb{Z} / p \mathbb{Z}) . "$ To appear in J. Eur. Math. Soc., preprint available at: arxiv.org:0807.2027.

[MCC] Chang, M.-C. "Product theorems in $\mathrm{SL}_{2}$ and $\mathrm{SL}_{3} . "$ J. Math. Jussieu. 7 (2008): 1-25.

[PS] Pyber, L., and E. Szabó. "Growth in finite simple groups of Lie type." Preprint available at: arxiv.org:1001.4556.

[V] Varjú, P., "Expansion in $\mathrm{SL}_{d}\left(\mathscr{O}_{K} / I\right), I$ squarefree." Preprint.

School of Mathematics, University of Bristol, Bristol, BS8 1TW, United Kingdom 